\title{
Travelling waves in a singularly perturbed sine-Gordon equation
}

\author{
Gianne Derks \\ Department of Mathematics and Statistics \\ University of Surrey, Guildford, Surrey, GU2 7XH \\ Email: G.Derks@surrey.ac.uk \\ Arjen Doelman \\ Faculty of Science \\ University of Amsterdam \\ Email: Doelman@science.uva.nl \\ Stephan van Gils and Timco Visser \\ Department of Applied Mathematics \\ University of Twente \\ P.O. Box 217, 7500 AE Enschede \\ The Netherlands \\ Email: Stephan@math.utwente.nl, Timco@math.utwente.nl
}

November 12, 2002

\begin{abstract}
We determine the linearised stability of travelling front solutions of a perturbed sineGordon equation. This equation models the long Josephson junction using the RCSJ model for currents across the junction and includes surface resistance for currents along the junction. The travelling waves correspond to the socalled fluxons and their linear stability is determined by calculating the Evans function. Surface resistance corresponds to a singular perturbation term in the governing equation, which specifically complicates the computation of the corresponding Evans function. Both the flow of quasi-particles across and along the junction stabilise the waves.
\end{abstract}

\section{Introduction}

Consider the following perturbed sine-Gordon equation

$$
u_{t t}-u_{x x}+\sin u=\varepsilon\left(\gamma-\alpha u_{t}+\beta u_{x x t}\right),
$$

where $\varepsilon \geq 0$ for definiteness. This equation models the long Josephson junction: two superconductors separated by a thin insulating layer, see for instance [23] and [3] and the references given there. Here we consider one direction (the $x$-direction) large compared to the Josephson penetration depth. In case $\varepsilon=0,(1.1)$ reduces to the sine-Gordon equation, which has a Hamiltonian structure and which is completely integrable. Physically this means that 
the superconductors are ideal, and there are no quasi-particle currents. All electrons have condensed to the superconducting states; they have all formed Cooper pairs. The electrons of the two superconductors can be described by a single wave function, and $u$ denotes the phase difference of the electrons between the top and the bottom superconductor.

The sine-Gordon equation can be obtained using the CSJ-model, in which it is assumed that across the junction there is only a supercurrent and a current due to capacitive effects and an inductance for currents along the junction, see $[9,26]$. The right hand side of equation (1.1) consists of three terms. The first one models the applied biased current, which is assumed to be uniform. The second one models the tunnelling of quasi-particles using the RCSJ model for currents across the junction. The third term models the flow of quasi-particles in the superconductors. It involves a third order derivative, and therefore it is a singular pertubation, which is interesting from mathematical point of view. This flow leads to an energy loss comparable to surface resistance. The energy loss due to surface resistance and tunneling depends on the material, and is small in practice. This justifies the rescaling of these material parameters by $\varepsilon$. The applied biased current $\gamma$ does not need to be small and is here taken small to be able to perform a perturbation analysis.

A fluxon corresponds to a travelling front solution with an increase of the phase $u$ by $2 \pi$. The sine-Gordon equation has a family of travelling wave front (kink or soliton) solutions which travel left or right with wave speed $(c)$ less than 1. For $\varepsilon>0$ small, the perturbed equation also admits front solutions, but the wave speed is no longer free, but selected by the parameter values.

The driving force for physics to research the long junction, are the various possible applications $[14,27,21]$. In all these applications, the existence of fluxons play an important role. Fluxons are current vortices that can travel along the junction. For this reason the study of fluxons and their stability has been a topic of research for a long time $[19,20,25]$. In most cases the behaviour of the fluxons is predicted using perturbation theory. In the present paper we will give a more rigorous treatment of both the existence of the fluxons, and the stability thereof. Existence results for perturbed fluxons based on energy methods are given in [18]. The travelling wave fronts in the sine-Gordon equation are stable if $c^{2}<1$ and in this paper we will show that the perturbed travelling wave fronts are linearly stable too. When $\beta=0$, this immediately gives nonlinear stability due to a result in [2].

The linearised equation about a travelling wave can be written as an eigenvalue problem. By using the complete integrability of the sine-Gordon equation, it is possible to find the general solution of the linearisation of the sine-Gordon equation about the kink solution, see MANN [17]. From this it follows that the unperturbed linearised sine-Gordon equation has a double eigenvalue at zero and continuous spectrum on the imaginary axis. So for $\varepsilon>0$ small, the perturbed linearised sine-Gordon equation can have eigenvalues near zero or near the essential spectrum.

To study the behaviour of the eigenvalues we will use the Evans function. The Evans function is a complex valued function with the property that to the right of the continuous spectrum the zeros of this function correspond to isolated eigenvalues of the linearised system. In its original definition, the Evans function is not defined near the essential spectrum but in the literature there have been several extensions of the Evans function to deal with this problem. We will use an extension close to the work of Kapitula \& Sandstede [11] and GARDNER \& Zumbrun [8].

The results presented in this paper can also be seen as a first step towards a mathematical 
understanding of the dynamics of fluxons in long Josephson junctions. Here, we consider the simplest problem of a single fluxon in one junction. In the physics literature, the main interest lies in the dynamics and interactions of various types of fluxons, or periodic 'trains' of fluxons, in multi-layered 'stacks' of long Josephson junctions. The methods developed in this paper can and will be extended, so that these more complex phenomena can also be analysed and understood mathematically.

In section 2 we analyse the persistence of the fluxons in the perturbed equation, followed by a formulation of the spectral problem in section 3 . Related to the spectral problem is the Evans function. In section 4 we analyse this function when surface resistance is absent, i.e. when $\beta=0$. In section 5 we include a small surface resistance. This leads to a singular ODE. However, when looking for the stability of the travelling waves, we find that the effects of the perturbation are eventually similar to the regular case.

\section{Existence of the travelling wave solutions}

The family of travelling wave front (kink) solutions of the sine-Gordon equation $(\varepsilon=0)$ consists of waves of the form

$$
u_{0}(x, t)=4 \arctan \left(e^{\xi}\right), \quad \text { where } \quad \xi=\frac{x-c t+x_{0}}{\sqrt{1-c^{2}}},
$$

for $|c|<1$ (see also Remark 2.5). To simplify notation, we define a coordinate transformation $\xi=\frac{x-c t+x_{0}}{\sqrt{1-c^{2}}}$ and new variables $\widetilde{u}(\xi, t)$ such that $u(x, t)=\widetilde{u}(\xi, t)$. Dropping the tildes we arrive at the equation

$$
u_{t t}-2 \frac{c}{\sqrt{1-c^{2}}} u_{\xi t}-u_{\xi \xi}+\sin u=\varepsilon\left(\gamma+\frac{\alpha c}{\sqrt{1-c^{2}}} u_{\xi}-\alpha u_{t}-\frac{\beta c}{\left(\sqrt{1-c^{2}}\right)^{3}} u_{\xi \xi \xi}+\frac{\beta}{1-c^{2}} u_{\xi \xi t}\right)
$$

The travelling wave equation for the perturbed system is

$$
u_{\xi \xi}-\sin u=-\varepsilon\left(\gamma+\frac{\alpha c}{\sqrt{1-c^{2}}} u_{\xi}-\frac{\beta c}{\left(\sqrt{1-c^{2}}\right)^{3}} u_{\xi \xi \xi}\right) .
$$

Theorem 2.1 Let $\alpha>0, \beta \geq 0$ and $\gamma \in \mathbb{R}$ be fixed. There exists a unique smooth curve of parameters $\varepsilon \mapsto c_{\varepsilon}$ and a unique smooth curve of wave shapes $\varepsilon \mapsto u_{\varepsilon}(\cdot)$ such that for $\varepsilon$ small, the waves $u_{\varepsilon}(\xi)$ are travelling wave solutions of $(2.2)$ with $c=c_{\varepsilon}$ and $u_{\varepsilon}(0)=\pi$. Furthermore, in the limit for $\varepsilon \rightarrow 0$, we have

$$
u_{0}(\xi)=4 \arctan \left(e^{\xi}\right), \quad \xi=\frac{x-c_{0} t+x_{0}}{\sqrt{1-c_{0}^{2}}}
$$

and $c_{0}$ is a solution of the equation

$$
3 \pi \gamma\left(\sqrt{1-c^{2}}\right)^{3}+4 c\left[3 \alpha\left(1-c^{2}\right)+\beta\right]=0 .
$$

Remark 2.2 Relation (2.3) implies that $c_{0}$ and $\gamma$ have opposite signs. Furthermore, it is not difficult to prove that for positive values of $\alpha, \beta$ and $\gamma$ equation (2.3) has one solution for $c$, denoted by $c_{0}$. 


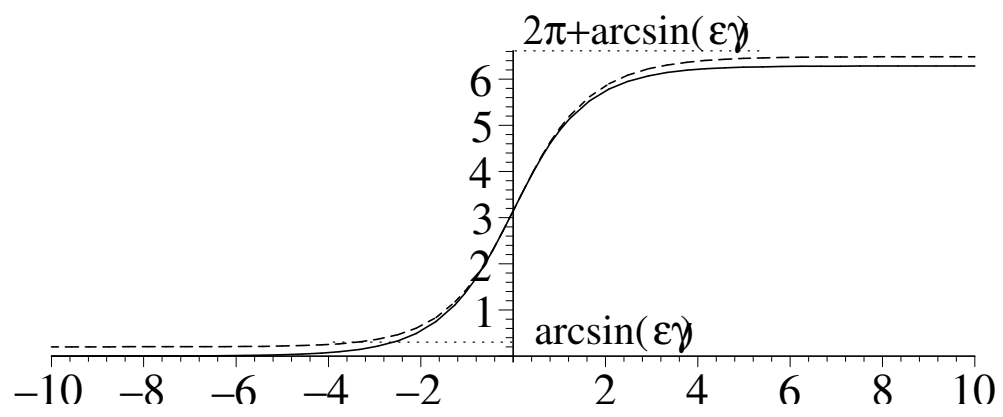

Figure 1: The unperturbed (unbroken line) and the perturbed travelling wave (dashed line).

In Figure 1, we have sketched $u_{\varepsilon}$ in case $\beta=0$. Note the limits for $\xi \rightarrow \pm \infty$.

Proof of Theorem 2.1 For $c \beta \neq 0$, the travelling wave equation (2.2) is a singularly perturbed problem. By introducing the coordinates $v=u_{\xi}, w=v_{\xi}=u_{\xi \xi}$, we can write the travelling wave equation as the (slow) system

$$
\begin{aligned}
u_{\xi} & =v \\
v_{\xi} & =w \\
\frac{\varepsilon \beta c}{\left(\sqrt{1-c^{2}}\right)^{3}} w_{\xi} & =w-\sin u+\varepsilon\left(\gamma+\frac{\alpha c}{\sqrt{1-c^{2}}} v\right)
\end{aligned}
$$

Introducing the fast variable $\zeta$ as $\varepsilon \zeta=\xi$, we can write the travelling wave equation as the fast system

$$
\begin{aligned}
u_{\zeta} & =\varepsilon v \\
v_{\zeta} & =\varepsilon w \\
\frac{\beta c}{\left(\sqrt{1-c^{2}}\right)^{3}} w_{\zeta} & =w-\sin u+\varepsilon\left(\gamma+\frac{\alpha c}{\sqrt{1-c^{2}}} v\right)
\end{aligned}
$$

The asymptotic $(\varepsilon=0)$ slow system is

$$
\left\{\begin{array} { r l } 
{ u _ { \xi } } & { = v } \\
{ v _ { \xi } } & { = w } \\
{ 0 } & { = w - \operatorname { s i n } u }
\end{array} \quad \text { hence } \quad \left\{\begin{array}{rl}
u_{\xi \xi} & =\sin u \\
v & =u_{\xi} \\
w & =\sin u
\end{array}\right.\right.
$$

Heteroclinic solutions of this system correspond to the travelling wave solutions of the unperturbed sine-Gordon equation: $u_{0}(\xi)=4 \arctan \left(e^{\xi}\right)$. The asymptotic fast system is

$$
\left\{\begin{array} { r l } 
{ u _ { \zeta } } & { = 0 } \\
{ v _ { \zeta } } & { = 0 } \\
{ \frac { \beta c } { ( \sqrt { 1 - c ^ { 2 } } ) ^ { 3 } } w _ { \zeta } } & { = w - \operatorname { s i n } u }
\end{array} \quad \text { hence } \quad \left\{\begin{array}{rl}
u & =u_{0} \\
v & =v_{0} \\
\frac{\beta c}{\left(\sqrt{1-c^{2}}\right)^{3}} w_{\zeta} & =w-\sin u_{0}
\end{array}\right.\right.
$$

Thus, the critical points (slow manifold) of the system are $w_{0}=\sin u_{0}$, which are attracting if $\beta c<0$. This manifold is also clearly normally hyperbolic if $\beta c \neq 0$. So for $\varepsilon$ small and $\beta c \neq 0$, Fenichel's Theorem [7] gives the existence of an invariant (center) manifold

$$
w=h(u, v, \varepsilon)=\sin u+\varepsilon h_{1}(u, v, \varepsilon),
$$


where $h_{1}(u, v, \varepsilon)$ satisfies

$$
h_{1}(u, v, \varepsilon)+\gamma+\frac{\alpha c}{\sqrt{1-c^{2}}} v-\frac{\beta c}{\left(\sqrt{1-c^{2}}\right)^{3}} v \cos u=\frac{\varepsilon \beta c}{\left(\sqrt{1-c^{2}}\right)^{3}}\left(\frac{\partial h_{1}}{\partial u} v+\left(\sin u+\varepsilon h_{1}\right) \frac{\partial h_{1}}{\partial v}\right) .
$$

Hence in lowest order,

$$
h_{1}(u, v, 0)=\left(\frac{\beta c}{\left(\sqrt{1-c^{2}}\right)^{3}} \cos u-\frac{\alpha c}{\sqrt{1-c^{2}}}\right) v-\gamma .
$$

The dynamics on the slow manifold is given by

$$
\begin{aligned}
& u_{\xi}=v \\
& v_{\xi}=\sin u+\varepsilon h_{1}(u, v, 0)+\mathcal{O}\left(\varepsilon^{2}\right)
\end{aligned}
$$

This is a perturbed Hamiltonian system with Hamiltonian $H(u, v)=\frac{1}{2} v^{2}+\cos u$ and nonHamiltonian perturbation $G(u, v, \varepsilon)=\varepsilon\left(0, h_{1}(u, v, 0)\right)+\mathcal{O}\left(\varepsilon^{2}\right)$. For $\varepsilon=0$, the heteroclinic solution is given by $u_{0}(\xi)=4 \arctan \left(e^{\xi}\right)$ and $v_{0}(\xi)=u_{0}^{\prime}(\xi)=2 \operatorname{sech}(\xi)$. The Melnikov function for the perturbed system is

$$
M(\varepsilon)=\int_{-\infty}^{\infty} \nabla H\left(u_{0}(\xi)\right) \cdot G\left(u_{0}(\xi), v_{0}(\xi), 0\right) d \xi+\mathcal{O}(\varepsilon) .
$$

Thus

$$
\begin{aligned}
M(0) & =-\int_{-\infty}^{\infty} \gamma v_{0}(\xi)+\left(\frac{\alpha c}{\sqrt{1-c^{2}}}-\frac{\beta c}{\left(\sqrt{1-c^{2}}\right)^{3}} \cos u_{0}(\xi)\right) v_{0}(\xi)^{2} d \xi \\
& =-2 \pi \gamma-\frac{8}{3} \frac{\beta c}{\left(\sqrt{1-c^{2}}\right)^{3}}-8 \frac{\alpha c}{\sqrt{1-c^{2}}} .
\end{aligned}
$$

Hence the solvability condition for the existence of a heteroclinic connection between the fixed points $\arcsin (\varepsilon \gamma)$ and $2 \pi+\arcsin (\varepsilon \gamma)$ is $M(\varepsilon)=0$. The lowest order of this equation implies that

$$
3 \pi \gamma\left(\sqrt{1-c^{2}}\right)^{3}+4 c\left(3 \alpha\left(1-c^{2}\right)+\beta\right)=0 .
$$

This gives the existence of a curve of heteroclinic connections and a curve of parameters as specified in the theorem, if $\beta c \neq 0$ (see GuCKEnheimer \& Holmes [10]). We select one travelling wave solution by imposing the condition $u_{\varepsilon}(0)=\pi$, to get rid of the arbitrary phase-shift.

Equation (2.2) is no longer singularly perturbed when $\beta c=0$. Hence the dynamics is two-dimensional and is given by $(2.6)$ with $h_{1}(u, v, 0)=-\gamma-\frac{\alpha c}{\sqrt{1-c^{2}}} v$. A Melnikov argument, simular to the one before gives the existence of a travelling wave solution and the expression for the wave speed as given in the Theorem with $\beta c=0$.

Using symmetry of the travelling wave equation, it can be shown that the travelling wave solutions and its derivatives with respect to $\varepsilon$ are either even or odd and that the wave speed $c_{\varepsilon}$ is a function of $\varepsilon^{2}$.

Lemma 2.3 Let $\alpha>0, \beta \geq 0$ and $\gamma \in \mathbb{R}$ be fixed. Then for $\varepsilon$ small and $\xi \in \mathbb{R}$ we have

$$
u_{-\varepsilon}(-\xi)=2 \pi-u_{\varepsilon}(\xi) \text { and } c_{-\varepsilon}=c_{\varepsilon} .
$$

This implies that for any $k \in \mathbb{N}$, the functions $\left.\frac{\partial^{k} u_{\varepsilon}}{\partial \varepsilon^{k}}\right|_{\varepsilon=0}(\xi)$ are even in $\xi$ for $k$ odd and odd in $\xi$ for $k$ even. Furthermore, $c_{\varepsilon}$ is a function of $\varepsilon^{2}$. 
Proof. Define the wave $v_{\varepsilon}(\xi)=2 \pi-u_{-\varepsilon}(-\xi)$. Then its derivatives satisfy $\left(\left(\frac{\mathrm{d}}{\mathrm{d} \xi}\right)^{k} v_{\varepsilon}\right)(\xi)=$ $(-1)^{k+1}\left(\left(\frac{\mathrm{d}}{\mathrm{d} \xi}\right)^{k} u_{-\varepsilon}\right)(-\xi)$ for any $k \in \mathbb{N}$ and $v_{\varepsilon}(0)=2 \pi-u_{\varepsilon}(0)=\pi$. Since the pair $\left(c_{\varepsilon}, u_{\varepsilon}(\xi)\right)$ is a solution of the travelling wave equation $(2.2)$, we get that $v_{\varepsilon}(\xi)$ is a solution of

$$
\begin{aligned}
\left(v_{\varepsilon}\right)_{\xi \xi}(\xi) & =-\left(u_{-\varepsilon}\right)_{\xi \xi}(-\xi)=-\left[\sin \left(u_{-\varepsilon}\right)+\varepsilon\left(\gamma+\frac{\alpha c_{-\varepsilon}}{\sqrt{1-c_{-\varepsilon}^{2}}}\left(u_{-\varepsilon}\right)_{\xi}-\frac{\beta c_{-\varepsilon}}{\left(\sqrt{1-c_{-\varepsilon}^{2}}\right)^{3}}\left(u_{-\varepsilon}\right)_{\xi \xi \xi}\right)\right](-\xi) \\
& =\sin \left(v_{\varepsilon}(\xi)\right)-\varepsilon\left(\gamma+\frac{\alpha c_{-\varepsilon}}{\sqrt{1-c_{-\varepsilon}^{2}}}\left(v_{\varepsilon}\right)_{\xi}(\xi)-\frac{\beta c_{-\varepsilon}}{\left(\sqrt{1-c_{-\varepsilon}^{2}}\right)^{3}}\left(v_{\varepsilon}\right)_{\xi \xi \xi}(\xi)\right)
\end{aligned}
$$

In other words, $v_{\varepsilon}(\xi)$ is a solution of the travelling wave equation (2.2) with $c=c_{-\varepsilon}$ and $v_{\varepsilon}(0)=\pi$. Since the travelling wave pair $\left(c_{\varepsilon}, u_{\varepsilon}\right)$ with $u_{\varepsilon}(0)=\pi$ is unique, this implies that $v_{\varepsilon}=u_{\varepsilon}$ and $c_{-\varepsilon}=c_{\varepsilon}$.

It is also possible to obtain expressions for the higher order terms in the expansion for the travelling wave solution $u_{\varepsilon}$. We write

$$
u_{\varepsilon}(\xi)=u_{0}(\xi)+\varepsilon u_{1}(\xi)+\varepsilon^{2} u_{2}(\xi)+\mathcal{O}\left(\varepsilon^{3}\right) .
$$

It follows from the travelling wave equation (2.2) that $\lim _{\xi \rightarrow-\infty} u_{\varepsilon}(\xi)=\arcsin \varepsilon \gamma$ and $\lim _{\xi \rightarrow \infty} u_{\varepsilon}(\xi)=$ $2 \pi+\arcsin \varepsilon \gamma$. Equation (2.6) gives that the equation for $u_{1}$ is of the form

$$
\left(u_{1}\right)_{\xi \xi}-\cos \left(u_{0}(\xi)\right) u_{1}=-\gamma-\frac{\alpha c_{0}}{\sqrt{1-c_{0}^{2}}}\left(u_{0}\right)_{\xi}(\xi)+\frac{\beta c_{0}}{\left(\sqrt{1-c_{0}^{2}}\right)^{3}} \cos \left(u_{0}(\xi)\right) .
$$

Also the equations for the higher order terms $u_{i}(\xi)$ will be of the form $\left(u_{i}\right)_{\xi \xi}-\cos \left(u_{0}(\xi)\right) u_{i}=$ $f_{i}(\xi)$, where $f_{i}(\xi)$ is a function of the lower order terms. Expressions for solutions of this type of equation can be determined with the Lemma below.

Lemma 2.4 Assume that the function $f(\xi)$ satisfies

$$
\int_{-\infty}^{\infty} f(\xi) \operatorname{sech} \xi d \xi=0
$$

Then the equation $u_{\xi \xi}-\cos \left(u_{0}(\xi)\right) u=f(\xi)$ has a bounded solution on $\mathbb{R}$. If the condition (2.7) is satisfied, then the unique solution satisfying $u(0)=0$ is given by

$$
\left(\begin{array}{c}
u(\xi) \\
u_{\xi}(\xi)
\end{array}\right)=X(\xi)\left[\int_{-\infty}^{\xi} P^{u} X^{-1}(\tau) F(\tau) d \tau+\int_{0}^{\xi} P^{s} X^{-1} F(\tau) d \tau\right]
$$

where

$X(\xi)=\left[\begin{array}{cc}\operatorname{sech} \xi & \frac{1}{2}(\xi \operatorname{sech} \xi+\sinh \xi) \\ (\operatorname{sech} \xi)^{\prime} & \frac{1}{2}(\xi \operatorname{sech} \xi+\sinh \xi)^{\prime}\end{array}\right], P^{u}=\left[\begin{array}{ll}0 & 0 \\ 0 & 1\end{array}\right], P^{s}=I-P^{u}$ and $F(\tau)=\left[\begin{array}{c}0 \\ f(\tau)\end{array}\right]$.

Proof. Writing $U=\left(u, u_{\xi}\right)^{T}$, we obtain for $U$ the first order equation

$$
\frac{d}{d \xi} U=A(\xi) U+F(\xi)
$$


where $A(\xi)=\left[\begin{array}{cc}0 & 1 \\ \cos \left(u_{0}(\xi)\right) & 0\end{array}\right]$. The matrix $X(\xi)$ is a solution matrix of the linear equation $\frac{d}{d \xi} U=A(\xi) U$. We will construct the solution $U$ of (2.9) as the intersection of the stable and the unstable manifold. Since $P^{u}$ is the projection on the unstable manifold at $\xi=0,(2.9)$ has a bounded solution on $\mathbb{R}_{+}$provided

$$
P^{u} U(0)=\int_{\infty}^{0} P^{u} X^{-1}(\tau) F(\tau) d \tau
$$

Similarly, since $\widehat{P}^{s}=P^{u}$ is the projection on the stable manifold at $\xi=0,(2.9)$ has a bounded solution on $\mathbb{R}$ provided

$$
\widehat{P}^{s} U(0)=\int_{-\infty}^{0} \widehat{P}^{s} X^{-1}(\tau) F(\tau) d \tau .
$$

As $P^{u}=\widehat{P}^{s}$, this is equivalent to the solvability condition on $f$. The explicit representation for $U$ follows immediately, since $u(0)=0$ is equivalent to the condition $P^{s} U(0)=0$.

By applying the Lemma to $f(\xi)=-\gamma-\frac{\alpha c_{0}}{\sqrt{1-c_{0}^{2}}}\left(u_{0}\right)_{\xi}(\xi)+\frac{\beta c_{0}}{\left(\sqrt{1-c_{0}^{2}}\right)^{3}} \cos \left(u_{0}(\xi)\right)$ an expression for $u_{1}$ can be obtained. First we note that the condition (2.7) on $f$ in the Lemma corresponds to the solvability condition given by the Melnikov function, since $v_{0}=\left(u_{0}\right)_{\xi}=2 \operatorname{sech} \xi$. Hence this condition is satisfied by the definition of $c_{0}$, as given in Theorem 2.1. For later use, we work out equation (2.8) in case $\beta=0$ and obtain the following identity

$$
\begin{aligned}
u_{1}(\xi)= & \gamma \operatorname{sech}(\xi)\left[\frac{\left(e^{\xi}-1\right)^{2}}{e^{2 \xi}+1}-\frac{\pi \xi\left(e^{2 \xi}-1\right)}{4\left(e^{2 \xi}+1\right)}+\int_{0}^{\xi} \frac{8 \tau e^{3 \tau}}{\left(1+e^{2 \tau}\right)^{3}} d \tau\right]+ \\
& \gamma \frac{(\xi \operatorname{sech} \xi+\sinh \xi)}{2}\left[\frac{\pi e^{2 \xi}}{e^{2 \xi}+1}-\frac{2\left(e^{\xi}-e^{3 \xi}\right)}{\left(e^{2 \xi}+1\right)^{2}}-2 \arctan e^{\xi}\right]
\end{aligned}
$$

This function is clearly even as predicted by Lemma 2.3 .

Remark 2.5 The (unperturbed or perturbed) sine-Gordon equation also has solutions that travel with speed $|c|>1$. However, these waves correspond to heteroclinic orbits that connect the trivial states $u \equiv \mp \pi$ to $u \equiv \pm \pi$ (at leading order). These states are unstable, so that these waves are irrelevant for the dynamics of (1.1).

\section{The spectral problem and the Evans function}

First we look at the linear stability of the travelling wave solutions which we have found in the previous section. The linear stability problem leads to a spectral problem and we will analyse this problem by using a so-called Evans function. The linearisation about the travelling wave solution $u_{\varepsilon}(\xi)$ of the perturbed sine-Gordon equation gives

$$
\begin{aligned}
v_{t t}-v_{\xi \xi}-\frac{2 c_{\varepsilon}}{\sqrt{1-c_{\varepsilon}^{2}}} & v_{\xi t}+\cos \left(u_{\varepsilon}\right) v= \\
& \varepsilon\left(\frac{\alpha c_{\varepsilon}}{\sqrt{1-c_{\varepsilon}^{2}}} v_{\xi}-\alpha v_{t}-\frac{\beta c_{\varepsilon}}{\left(\sqrt{1-c_{\varepsilon}^{2}}\right)^{3}} v_{\xi \xi \xi}+\frac{\beta}{1-c_{\varepsilon}^{2}} v_{\xi \xi t}\right) .
\end{aligned}
$$


The spectral Ansatz $v(\xi, t)=e^{\lambda t} \widehat{v}(\xi)$ leads to the following spectral problem

$$
\begin{aligned}
\left(\lambda^{2}+\cos \left(u_{\varepsilon}\right)\right) \widehat{v}- & \widehat{v}_{\xi \xi}-\frac{2 \lambda c_{\varepsilon}}{\sqrt{1-c_{\varepsilon}^{2}}} \widehat{v}_{\xi}= \\
& \quad\left(\frac{\alpha c_{\varepsilon}}{\sqrt{1-c_{\varepsilon}^{2}}} \widehat{v}_{\xi}-\alpha \lambda \widehat{v}-\frac{\beta c_{\varepsilon}}{\left(\sqrt{1-c_{\varepsilon}^{2}}\right)^{3}} \widehat{v}_{\xi \xi \xi}+\frac{\beta \lambda}{1-c_{\varepsilon}^{2}} \widehat{v}_{\xi \xi}\right) .
\end{aligned}
$$

Like the existence problem, of course the linear stability problem has a singularly perturbed nature for $\beta \neq 0$ too. We will distinguish throughout this analysis between the regular case $(\beta=0)$ and the singular case $(\beta \neq 0)$.

The analysis of the regular case is similar to that of [11], mainly because the sine-Gordon equation shares many relevant features with the nonlinear Schrödinger equation. It is known that singularly perturbed linear stability problems may exhibit subtle, unexpected phenomena. For instance, small (but singular) perturbations may have a leading order influence on the eigenvalues (see $[5,6]$ ). Therefore, a careful analysis of the full, singular problem is necessary. The special, regular, case $\beta=0$ serves as a firm foundation of the singular analysis.

Remark 3.1 The analysis in the subsequent sections is mostly devoted to the analysis of the Evans function(s) near $\lambda=0$ and near the 'edge' of the essential spectrum $\lambda= \pm i \omega_{0}$. The local analysis of the Evans function near $\lambda=0$ has been put in a general theoretical framework in [12]. The possibility of eigenvalues appearing from the edge of the essential spectrum in a general setting of nearly integrable systems has been studied in [13], again by

Evans function techniques. However, neither of these papers consider the effects of higher order, i.e. singular, perturbations.

\subsection{The Evans function without surface resistance}

In this section we will focus on the non-singular case. When $\beta=0$, we rewrite (3.2) as a first order system by introducing $U(\xi)=\left(\widehat{v}(\xi), \widehat{v}_{\xi}(\xi)\right)^{T}$. This gives the linear equation

$$
U_{\xi}=\mathbf{A}(\xi, \lambda, \varepsilon) U
$$

where

$$
\mathbf{A}(\xi, \lambda, \varepsilon)=\left(\begin{array}{cc}
0 & 1 \\
\lambda^{2}+\alpha \varepsilon \lambda+\cos \left(u_{\varepsilon}\right) & -\frac{c_{\varepsilon}}{\sqrt{1-c_{\varepsilon}^{2}}}(2 \lambda+\varepsilon \alpha)
\end{array}\right) .
$$

Note that this system is (non-autonomous) Hamiltonian if $\lambda=0$ and $\varepsilon=0$.

The front solutions decay exponentially fast to $\arcsin (\varepsilon \gamma)$ and $\arcsin (\varepsilon \gamma)+2 \pi$ at $x=\mp \infty$ respectively. So we can define the system at infinity (far field equations) as

$$
U_{\xi}=\mathbf{A}_{0}(\lambda, \varepsilon) U, \quad \text { where } \quad \mathbf{A}_{0}(\lambda, \varepsilon)=\lim _{|\xi| \rightarrow \infty} \mathbf{A}(\xi, \lambda, \varepsilon) .
$$

Note that this a linear autonomous ODE. Since $\lim _{|\xi| \rightarrow \infty} \cos \left(u_{\varepsilon}(\xi)\right)=\sqrt{1-\varepsilon^{2} \gamma^{2}}$, the characteristic polynomial of $\mathbf{A}_{0}$ is

$$
p(\mu, \lambda, \varepsilon)=\mu^{2}+\frac{c_{\varepsilon} \mu}{\sqrt{1-c_{\varepsilon}^{2}}}(2 \lambda+\varepsilon \alpha)-\left(\lambda^{2}+\lambda \varepsilon \alpha+\sqrt{1-\varepsilon^{2} \gamma^{2}}\right) .
$$


This shows that $\mathbf{A}_{0}$ has double eigenvalues for $\lambda=-\frac{\varepsilon \alpha}{2} \pm i \omega_{\varepsilon}$, with

$$
\omega_{\varepsilon}^{2}=\left(1-c_{\varepsilon}^{2}\right)\left(\sqrt{1-\varepsilon^{2} \gamma^{2}}-\varepsilon^{2} \alpha^{2} / 4\right) .
$$

In Figure 2, we have sketched the eigenvalues of $\mathbf{A}_{0}$ for $\lambda \in \mathbb{C}$. At the two places in the $\lambda$-plane, where $\mathbf{A}_{0}$ has a double eigenvalue, we make cuts in the complex plane, denoted by the bold dashed lines in Figure 2. The bold solid lines are the continuous spectrum. In the

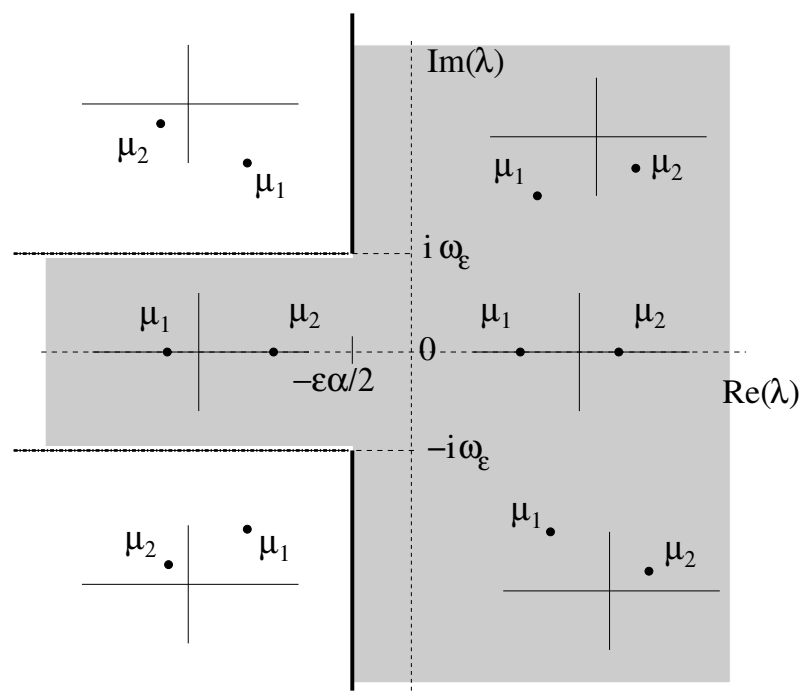

Figure 2: Eigenvalues of $\mathbf{A}_{0}$ for $\lambda \in \mathbb{C}$. Note the cuts (bold dashed lines) in the complex plane ending at $-\frac{\varepsilon \alpha}{2} \pm \mathrm{i} \omega_{\varepsilon}$. The bold solid lines are the continuous spectrum and the shaded region is denoted by $\Lambda_{\varepsilon}$.

$\lambda$-plane, we define the region

$$
\Lambda_{\varepsilon}=\left\{\lambda \in \mathbb{C} \mid \Re(\lambda)>-\frac{\varepsilon \alpha}{2} \quad \text { or } \quad|\Im(\lambda)|<\mathrm{i} \omega_{\varepsilon}\right\} .
$$

This region is shaded in Figure 2. For $\lambda \in \Lambda_{\varepsilon}$, there is an eigenvalue $\mu_{1}$ with negative real part and an eigenvalue $\mu_{2}$ with positive real part. So the linear system (3.3) has exactly one (up to scaling) solution which is exponentially decaying at $\xi=+\infty$, we denote this solution by $U^{+}(\xi, \lambda, \varepsilon)$ and one solution which is exponentially decaying at $\xi=-\infty$, we denote this solution by $U^{-}(\xi, \lambda, \varepsilon)$. The solutions $U^{ \pm}(\xi, \lambda, \varepsilon)$ can be chosen analytically in $\lambda$ for $\lambda \in \Lambda_{\varepsilon}$. For $\lambda \in \Lambda_{\varepsilon}$, the Evans function is an analytical function defined as

$$
D(\lambda, \varepsilon)=e^{-\int_{0}^{\xi} \operatorname{tr}(\mathbf{A}(s, \lambda, \varepsilon)) d s} U^{+}(\xi, \lambda, \varepsilon) \wedge U^{-}(\xi, \lambda, \varepsilon) .
$$

(cf. [1]). The Evans function can be made unique by using the normalisation

$$
\left[\lim _{\xi \rightarrow \infty} e^{-\mu_{1}(\lambda, \varepsilon) \xi} U^{+}(\xi, \lambda, \varepsilon)\right] \wedge\left[\lim _{\xi \rightarrow-\infty} e^{-\mu_{2}(\lambda, \varepsilon) \xi} U^{-}(\xi, \lambda, \varepsilon)\right]=1
$$

This normalisation has a number of advantages (see e.g. [4]), however, it degenerates near the endpoints of the essential spectrum (see Remark 3.5). The Evans function can also be defined uniquely by prescribing the behaviour of the solutions $U^{ \pm}(\xi, \lambda, \varepsilon)$ at $\xi= \pm \infty$ 
$[1,8,11,5,6]$. This method has as disadvantage that one needs explicit information on the linearised stability system at $\pm \infty$. However, this information can be determined for this problem: for any eigenvalue $\mu$, the eigenvector can be written as a multiple of $(1, \mu)^{T}$. Therefore, we normalise $D(\lambda, \varepsilon)$ by

$$
\lim _{\xi \rightarrow \infty} e^{-\mu_{1}(\lambda, \varepsilon) \xi} U^{+}(\xi, \lambda, \varepsilon)=\left(1, \mu_{1}\right)^{T} \quad \text { and } \quad \lim _{\xi \rightarrow-\infty} e^{-\mu_{2}(\lambda, \varepsilon) \xi} U^{-}(\xi, \lambda, \varepsilon)=\left(1, \mu_{2}\right)^{T} .
$$

In [11] it is shown how to extend the Evans function across the essential spectrum. Away from the branch points and the cuts, we continue the eigenvalues and eigenfunctions smoothly in a sufficiently small strip left of the essential spectrum while keeping the definition of $D$ the same. The resulting function is analytic in $\lambda$ and smooth in $\varepsilon$. We will come back to this in section 4.2. The extended Evans function equals the traditional definition for $\Re(\lambda)>0$. So eigenvalues with $\Re(\lambda)>0$ correspond with zeros of the Evans function in this region of the complex $\lambda$ plane.

The Evans function has some symmetry properties, which are induced by symmetry properties of the original system (3.1) and the travelling waves as given in Lemma 2.3.

Lemma 3.2 For $\lambda \in \Lambda_{\varepsilon}$, the Evans function satisfies

$$
D(\bar{\lambda}, \varepsilon)=\overline{D(\lambda, \varepsilon)} \quad \text { and } \quad D(-\lambda,-\varepsilon)=D(\lambda, \varepsilon) .
$$

Proof. First we observe that $\lambda$ is the only non-real element in the definition of $A(\xi, \lambda, \varepsilon)$, hence $\overline{A(\xi, \lambda, \varepsilon)}=A(\xi, \bar{\lambda}, \varepsilon)$. Together with the normalisation property, this implies that $D(\bar{\lambda}, \varepsilon)=\overline{D(\lambda, \varepsilon)}$.

Furthermore, using the symmetry properties of Lemma 2.3 for the travelling wave, we have that $\cos \left(u_{-\varepsilon}(-\xi)\right)=\cos \left(2 \pi-u_{\varepsilon}(\xi)\right)=\cos \left(u_{\varepsilon}(\xi)\right)$ and $c_{-\varepsilon}=c_{\varepsilon}$. Thus $\mathbf{A}(-\xi,-\lambda,-\varepsilon)=$ $-\mathbf{S A}(\xi, \lambda, \varepsilon) \mathbf{S}$, where $\mathbf{S}=\operatorname{diag}(1,-1)$. Using this, we get $D(-\lambda,-\varepsilon)=D(\lambda, \varepsilon)$.

Next, we determine the Evans function in case $\varepsilon=0$. In Mann [17], it is shown that the solutions of the linearised sine-Gordon equation (3.1), which can be related to the eigenvalue problem (3.2) with $\varepsilon=0$, are given by

$$
\begin{aligned}
v_{0}(\xi, t) & =\operatorname{sech} \xi \\
\tilde{v}_{0}(\xi, t) & =(2 \xi+\sinh (2 \xi)) \operatorname{sech} \xi=2(\xi \operatorname{sech} \xi+\sinh \xi) \\
v_{\lambda}^{ \pm}(\xi, t) & =e^{\lambda t} e^{\left(-b_{1} \pm b_{2}\right) \xi}\left(\tanh \xi \mp b_{2}\right), \quad b_{1}=\frac{\lambda c_{0}}{\sqrt{1-c_{0}^{2}}} \quad \text { and } \quad b_{2}=\frac{\sqrt{\lambda+\mathrm{i} \omega_{0}} \sqrt{\lambda-\mathrm{i} \omega_{0}}}{\sqrt{1-c_{0}^{2}}} \\
\tilde{v}_{i \omega_{0}}(\xi, t) & =(\xi \tanh \xi-1) e^{\mathrm{i}\left(\omega_{0} t-c_{0} \xi\right)}
\end{aligned}
$$

(recall that $\omega_{0}=\sqrt{1-c_{0}^{2}},(3.6)$ ). The first two solutions are the solutions at $\lambda=0$, the solutions $v_{\lambda}^{ \pm}$are valid for eigenvalues $\lambda \neq 0$. Note that $v_{\lambda}^{+}=v_{\lambda}^{-}$at $\lambda=\mathrm{i} \omega_{0}=\mathrm{i} \sqrt{1-c_{0}^{2}}$, thus the solution $\tilde{v}_{\mathrm{i} \omega_{0}}$ is the degenerate solution at $\lambda=\mathrm{i} \omega_{0}$.

Remark 3.3 Note that the linearised sine-Gordon equation (3.1) has more solutions than the ones mentioned above, for example $\widehat{v}_{0}(\xi, t)=\left(t \sqrt{1-c_{0}^{2}}-c_{0} \xi\right) \operatorname{sech}(\xi)$ is a solution. This solution can be found by differentiating $u_{0}(\xi)$ with respect to $c_{0}$, using the definition for $\xi$ as a function of $c_{0}, x$ and $t$. 
Based on (3.10), and incorporating the normalisation (3.9), we define

$$
\begin{aligned}
& U^{+}(\xi, \lambda, 0)=\frac{e^{-\left(b_{1}+b_{2}\right) \xi}}{1+b_{2}}\left(\left(b_{2}+\tanh \xi\right),-\left(b_{1}+b_{2}\right)\left(\tanh \xi+b_{2}\right)+\operatorname{sech}^{2} \xi\right)^{T} \\
& U^{-}(\xi, \lambda, 0)=\frac{e^{-\left(b_{1}-b_{2}\right) \xi}}{1+b_{2}}\left(\left(b_{2}-\tanh \xi\right),\left(b_{1}-b_{2}\right)\left(\tanh \xi-b_{2}\right)-\operatorname{sech}^{2} \xi\right)^{T}
\end{aligned}
$$

where we have used that $\mu_{1}(\lambda, 0)=-b_{1}-b_{2}$ and $\mu_{2}(\lambda, 0)=-b_{1}+b_{2}$. Note that $b_{2}$ has to be defined carefully to incorporate two cuts in the complex $\lambda$-plane. To be explicit,

$$
b_{2}=\frac{\sqrt{\lambda+\mathrm{i} \omega_{0}} \sqrt{\lambda-\mathrm{i} \omega_{0}}}{\sqrt{1-c_{0}^{2}}}, \quad \text { with } \quad \sqrt{z}=\sqrt{|z|} e^{\mathrm{i} \frac{\arg (z)}{2}}, \quad-\pi<\arg (z) \leq \pi .
$$

This leads to two horizontal cuts in the left half of the complex plane, ending at $\pm \mathrm{i} \omega_{0}$. Choosing the cuts in this way has the advantage that the symmetry properties as given in Lemma 3.2 are valid for all points in the complex plane, except at the cuts.

Lemma 3.4 When $\varepsilon=0$, the Evans function is explicitly given by the relation

$$
D(\lambda, 0)=-\frac{2 b_{2}\left(1-b_{2}\right)}{1+b_{2}}=2 \frac{\lambda^{2}+\omega_{0}^{2}-\omega_{0} \sqrt{\lambda+\mathrm{i} \omega_{0}} \sqrt{\lambda-\mathrm{i} \omega_{0}}}{\omega_{0}^{2}+\omega_{0} \sqrt{\lambda+\mathrm{i} \omega_{0}} \sqrt{\lambda-\mathrm{i} \omega_{0}}}
$$

Here the square roots are defined with branch cuts as in (3.12) and $\omega_{0}=\sqrt{1-c_{0}^{2}}$. The Evans function is real if $\lambda$ is real and has the asymptotic properties

$$
\begin{aligned}
& D(\lambda, 0)=\frac{\lambda^{2}}{2\left(1-c_{0}^{2}\right)}+\mathcal{O}\left(\lambda^{4}\right) \quad \text { for } \lambda \rightarrow 0, \\
& D(\lambda, 0)=-\frac{2(1 \pm \mathrm{i})}{\left(1-c_{0}^{2}\right)^{\frac{1}{4}}} \sqrt{\lambda \mp \mathrm{i} \omega_{0}}+\mathcal{O}\left(\lambda \mp \mathrm{i} \omega_{0}\right) \text { for } \lambda \rightarrow \pm i \omega_{0} \text {, } \\
& D(\lambda, 0)=\frac{2 \lambda}{\sqrt{1-c_{0}^{2}}}+\mathcal{O}\left(\frac{1}{\lambda}\right) \quad \text { for } \lambda \rightarrow \infty .
\end{aligned}
$$

Furthermore, at $\varepsilon=0$, the roots of the Evans function are at $\lambda=0$ (double) and at $\lambda= \pm \mathrm{i} \omega_{0}$. So these are the only points from which eigenvalues can bifurcate when $\varepsilon \neq 0$.

Proof. The Evans function at $\varepsilon=0$ follows from a direct computation using (3.11) and the fact that $\operatorname{tr}(\mathbf{A}(\xi, \lambda, 0))=-\frac{2 c_{0} \lambda}{\sqrt{1-c_{0}^{2}}}=-2 b_{1}(3.4)$.

Remark 3.5 The alternative normalisation (3.8) yields an additional factor $\frac{1}{2 b_{2}}$ in the Evans function. This factor is singular at $b_{2}=0$, i.e. at $\lambda= \pm \mathrm{i} \omega_{0}$. This is due to the fact that at these points the eigenvalues collide and the eigenspace becomes degenerate, i.e, there is only one eigenvector instead of two. At these two values of $\lambda$ the analytic definition of the alternative Evans function breaks down too, and they are the endpoints of the branch cuts. However, the Evans function itself is still well-defined at these points, but it does not vanish anymore.

\subsection{The Evans function for a small surface resistance}

In this section we will consider the singular case, i.e., $\beta \neq 0$. When $\beta \neq 0$, we rewrite the linearised equation (3.2) as a first order system by introducing $U(\xi)=\left(\widehat{v}(\xi), \widehat{v}_{\xi}(\xi), \widehat{v}_{\xi \xi}(\xi)\right)^{T}$. This gives the linear equation

$$
U_{\xi}=\mathbf{A}^{\beta}(\xi, \lambda, \varepsilon) U
$$


where

$$
\mathbf{A}^{\beta}(\xi, \lambda, \varepsilon)=\left(\begin{array}{ccc}
0 & 1 & 0 \\
0 & 0 & 1 \\
-\frac{\left(\lambda^{2}+\alpha \varepsilon \lambda+\cos \left(u_{\varepsilon}\right)\right)\left(\sqrt{1-c_{\varepsilon}^{2}}\right)^{3}}{\varepsilon \beta c_{\varepsilon}} & \frac{(2 \lambda+\varepsilon \alpha)\left(1-c_{\varepsilon}^{2}\right)}{\varepsilon \beta} & \frac{\left(1-c_{\varepsilon}^{2}+\varepsilon \beta \lambda\right) \sqrt{1-c_{\varepsilon}^{2}}}{\varepsilon \beta c_{\varepsilon}}
\end{array}\right) .
$$

The front solutions decay exponentially fast to $\arcsin (\varepsilon \gamma)$ and $\arcsin (\varepsilon \gamma)+2 \pi$ at $x=\mp \infty$ respectively. So we can define the system at infinity (far field equations) as

$$
U_{\xi}=\mathbf{A}_{0}^{\beta}(\lambda, \varepsilon) U, \quad \text { where } \quad \mathbf{A}_{0}^{\beta}(\lambda, \varepsilon)=\lim _{|\xi| \rightarrow \infty} \mathbf{A}^{\beta}(\xi, \lambda, \varepsilon) .
$$

Note that this a linear autonomous ODE. Since $\lim _{|\xi| \rightarrow \infty} \cos \left(u_{\varepsilon}(\xi)\right)=\sqrt{1-\varepsilon^{2} \gamma^{2}}$, the characteristic polynomial of $\mathbf{A}_{0}^{\beta}$ is

$$
p(\mu, \lambda, \varepsilon)=-\mu^{3}+\frac{\left(1-c_{\varepsilon}^{2}+\varepsilon \beta \lambda\right) \sqrt{1-c_{\varepsilon}^{2}}}{\varepsilon \beta c_{\varepsilon}} \mu^{2}+\frac{(2 \lambda+\varepsilon \alpha)\left(1-c_{\varepsilon}^{2}\right)}{\varepsilon \beta} \mu-\frac{\left(\lambda^{2}+\alpha \varepsilon \lambda+\sqrt{1-\varepsilon^{2} \gamma^{2}}\right)\left(\sqrt{1-c_{\varepsilon}^{2}}\right)^{3}}{\varepsilon \beta c_{\varepsilon}} .
$$

This shows that for $\varepsilon$ small, the matrix $\mathbf{A}_{0}^{\beta}$ has one large eigenvalue of order $\frac{1}{\varepsilon}$ and two order 1 eigenvalues, which correspond to the eigenvalues of the $\beta=0$ case. To be explicit, we have

$$
\begin{aligned}
& \mu_{1}(\lambda, \varepsilon)=-\frac{c_{\varepsilon} \lambda-\sqrt{-c_{\varepsilon}^{2}+\lambda^{2}+1}}{\sqrt{1-c_{\varepsilon}^{2}}}+\mathcal{O}(\varepsilon) \\
& \mu_{2}(\lambda, \varepsilon)=-\frac{c_{\varepsilon} \lambda+\sqrt{-c_{\varepsilon}^{2}+\lambda^{2}+1}}{\sqrt{1-c_{\varepsilon}^{2}}}+\mathcal{O}(\varepsilon) \\
& \mu_{3}(\lambda, \varepsilon)=\frac{\left(\sqrt{1-c_{\varepsilon}^{2}}\right)^{3}}{\varepsilon \beta c_{\varepsilon}}+\frac{\left(1+c_{\varepsilon}^{2}\right) \lambda}{c_{\varepsilon} \sqrt{1-c_{\varepsilon}^{2}}}+\varepsilon c_{\varepsilon} \frac{\alpha\left(1-c_{\varepsilon}^{2}\right)^{2}-\beta\left[\left(1-c_{\varepsilon}^{2}\right)+\lambda^{2}\left(c_{\varepsilon}^{2}+3\right)\right]}{\left(\sqrt{1-c_{\varepsilon}^{2}}\right)^{5}}+\mathcal{O}\left(\varepsilon^{2}\right) .
\end{aligned}
$$

The continuous spectrum is given by those $\lambda$ values which satisfy

$$
\left(\lambda \sqrt{1-c_{\varepsilon}^{2}}-\mathrm{i} c_{\varepsilon} \kappa\right)^{2}+\frac{\varepsilon}{\sqrt{1-c_{\varepsilon}^{2}}}\left(\alpha\left(1-c_{\varepsilon}^{2}\right)+\beta \kappa^{2}\right)\left(\lambda \sqrt{1-c_{\varepsilon}^{2}}-\mathrm{i} c_{\varepsilon} \kappa\right)+\kappa^{2}+\sqrt{1-\varepsilon^{2} \gamma^{2}} \sqrt{1-c_{\varepsilon}^{2}}=0
$$

for some $\kappa \in \mathbb{R}$. In Figure 3 a sketch of the continuous spectrum in case $\beta>0$ and $\varepsilon$ is small is given. Note that for $\beta \neq 0$, the continuous spectrum does not have endpoints near the imaginary axis, instead the continuous spectrum curves smoothly. This is due to the fact that the double eigenvalue has moved out of the continuous spectrum for $\beta \neq 0$ (see also section 5.3).

The expressions (3.16) for the eigenvalues $\mu_{i}$ imply that the far field equations have two slow solutions and one fast solution. In lowest order, the slow eigenvalues $\mu_{1}$ and $\mu_{2}$ are equal to the eigenvalues of the unperturbed linearised sine-Gordon equation. Hence for $\varepsilon$ small, there will be one slow positive eigenvalue, one slow negative eigenvalue and one fast eigenvalue, whose sign is equal to the sign of $\beta c_{\varepsilon}$.

We define the slow solutions $U^{-}(\xi, \lambda, \varepsilon)$ and $U^{+}(\xi, \lambda, \varepsilon)$ and the fast solution $U^{f}(\xi, \lambda, \varepsilon)$ to be such that they solve (3.13) and that $U^{+}(\xi, \lambda, \varepsilon)$ is exponentially decaying like $e^{\mu_{1} \xi}$ at $\xi=+\infty, U^{-}(\xi, \lambda, \varepsilon)$ is exponentially decaying like $e^{\mu_{2} \xi}$ at $\xi=-\infty$ and $U^{f}(\xi, \lambda, \varepsilon)$ is exponentially decaying like $e^{\mu_{3} \xi}$ at $\xi=-\operatorname{sgn}\left(\beta c_{\varepsilon}\right) \infty$. The Evans function is defined as

$$
D^{\beta}(\lambda, \varepsilon)=e^{-\int_{0}^{\xi} \operatorname{tr}\left(\mathbf{A}^{\beta}(s, \lambda, \varepsilon)\right) d s} U^{+}(\xi, \lambda, \varepsilon) \wedge U^{-}(\xi, \lambda, \varepsilon) \wedge U^{f}(\xi, \lambda, \varepsilon) .
$$




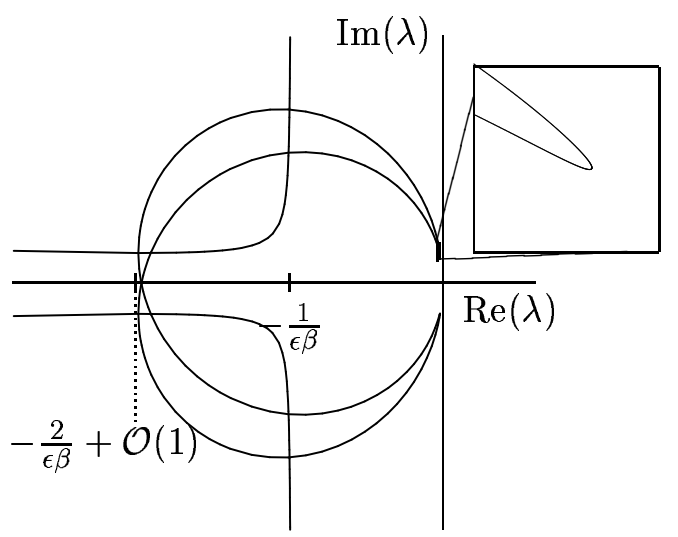

Figure 3: Sketch of the continuous spectrum for $\beta>0$ and $\varepsilon$ small. The picture on the right zooms in at the "corners" of the continuous spectrum near the imaginary axis and shows that the continuous spectrum does not have endpoints, but turns smoothly.

As before, we make the definition of the Evans function unique by prescribing the behaviour of $U^{+}, U^{-}$and $U^{f}$ at $\infty,-\infty$ and $-\operatorname{sgn}\left(\beta c_{\varepsilon}\right) \infty$,

$$
\begin{aligned}
\lim _{\xi \rightarrow \infty} e^{-\mu_{1}(\lambda, \varepsilon) \xi} U^{+}(\xi, \lambda, \varepsilon) & =\eta_{1}(\lambda, \varepsilon)=\left(1, \mu_{1}, \mu_{1}^{2}\right)^{T}, \\
\lim _{\xi \rightarrow-\infty} e^{-\mu_{2}(\lambda, \varepsilon) \xi} U^{-}(\xi, \lambda, \varepsilon) & =\eta_{2}(\lambda, \varepsilon)=\left(1, \mu_{2}, \mu_{1}^{3}\right)^{T}, \\
\lim _{\xi \rightarrow-\operatorname{sgn}\left(\beta c_{\varepsilon}\right) \infty} e^{-\mu_{3}(\lambda, \varepsilon) \xi} U^{f}(\xi, \lambda, \varepsilon) & =\eta_{3}(\lambda, \varepsilon)=\left(\frac{1}{\mu_{3}^{2}}, \frac{1}{\mu_{3}}, 1\right)^{T},
\end{aligned}
$$

where $\eta_{1,2,3}(\lambda, \varepsilon)$ are the eigenvectors associated to $\mu_{1,2,3}(\lambda, \varepsilon)$. As in the case without surface resistance, the Evans function has some symmetry properties induced by the symmetries of the original system. First we observe from the characteristic polynomial (3.15) that for $\varepsilon$ small a double eigenvalue occurs if the two slow eigenvalues collide, i.e., at

$$
\lambda= \pm \mathrm{i} \omega_{0}-\widetilde{\lambda}_{ \pm}(\varepsilon), \quad \text { where } \quad \widetilde{\lambda}_{+}(\varepsilon)=\overline{\widetilde{\lambda}_{-}(\varepsilon)}=\frac{\varepsilon}{2}\left(\alpha+\frac{\beta c_{0}^{2}}{1-c_{0}^{2}}\right)+\mathcal{O}\left(\varepsilon^{2}\right)
$$

Lemma 3.6 For $|\Re(\lambda)|<\Re(\widetilde{\lambda}(\varepsilon))$ or $|\Im(\lambda)|<\mathrm{i} \omega_{0}+\mathrm{i} \Im\left(\widetilde{\lambda}_{+}(\varepsilon)\right)$, the Evans function satisfies

$$
D^{\beta}(\bar{\lambda}, \varepsilon)=\overline{D^{\beta}(\lambda, \varepsilon)} \quad \text { and } \quad D^{\beta}(-\lambda,-\varepsilon)=D^{\beta}(\lambda, \varepsilon) .
$$

Proof. The proof is similar to the proof of Lemma 3.2 with $\mathbf{S}=\operatorname{diag}(1,-1,1)$.

In lowest order, the two slow solutions (corresponding to the order 1 eigenvalues) are related to the unperturbed sine-Gordon equation. Hence with $u^{+}(\xi, \lambda, 0)=e^{-\left(b_{1}+b_{2}\right) \xi}\left(b_{2}+\tanh \xi\right) /(1+$ $\left.b_{2}\right)$ and $u^{-}(\xi, \lambda, 0)=e^{-\left(b_{1}-b_{2}\right) \xi}\left(b_{2}-\tanh \xi\right) /\left(1+b_{2}\right)(3.10),(3.11)$ it follows that

$$
\begin{aligned}
& U^{+}(\xi, \lambda, 0)=\left(u^{+},\left(u^{+}\right)_{\xi},\left(u^{+}\right)_{\xi \xi}\right)(\xi, \lambda, 0) ; \\
& U^{-}(\xi, \lambda, 0)=\left(u^{-},\left(u^{-}\right)_{\xi},\left(u^{-}\right)_{\xi \xi}\right)(\xi, \lambda, 0)
\end{aligned}
$$

and $b_{1}$ and $b_{2}$ as given in (3.10). Looking at the lowest order in the fast system, we see that the fast solution satisfies

$$
\lim _{\varepsilon \rightarrow 0} e^{-\mu_{3}(\lambda, \varepsilon) \xi} U^{f}(\xi, \lambda, \varepsilon)=(0,0,1) .
$$


So altogether, in lowest order the Evans function for $\beta \neq 0$ is equal to the lowest order of the Evans function for $\beta=0$, i.e. the Evans function for the unperturbed linearised sine-Gordon system as given in Lemma 3.4 (with the same branch cuts as defined in (3.12)). So again, the only possibility for eigenvalues to bifurcate into the right half plane is from $\lambda=0$ or from the endpoints of the branch cuts $\lambda= \pm \mathrm{i} \omega_{0}$.

\section{Stability analysis with zero surface resistance}

In this section we will analyse the possible bifurcation of eigenvalues when $\beta=0$. This analysis shows that for $\alpha>0$, one of the double eigenvalues at $\lambda=0$ moves to the left. Furthermore, we will also show that there are no eigenvalues bifurcating out of the continuous spectrum. So, no eigenvalues bifurcate in the right half plane and hence the perturbed travelling wave solutions are linearly stable.

Theorem 4.1 In case of zero surface resistance $(\beta=0)$ and $\alpha>0$ the perturbed travelling wave $u_{\varepsilon}(\xi)$, with $\xi=\frac{x-c_{\varepsilon} t}{\sqrt{1-c_{\varepsilon}^{2}}}$, is spectrally stable for small values of $\varepsilon$.

Actually, linearized stability implies here also nonlinear stability. First we give a precise definition of orbital stability. For the details we refer to [2]. All the arguments given there for the Klein-Gordon equation carry over to the sine-Gordon equation.

Definition 4.2 Let $\beta=0$. The travelling wave solution $u_{\varepsilon}$ is an asymptotically stable travelling wave solution of (1.1) if there is a neighbourhood $N \subset H^{1} \times L^{2}$ of $\left(u_{\varepsilon}, 0\right)$ such that for any $(\hat{u}, \hat{v}) \in N$ there is a $k \in \mathbb{R}$ such that

$$
\left\|u(\cdot+k, t)-u_{\varepsilon}(\cdot)\right\|_{H_{1}}+\left\|u_{t}(\cdot+k, t)\right\|_{L_{2}} \rightarrow 0,
$$

as $t \rightarrow \infty$, where $u$ is the solution of (2.1) with $u(\cdot, 0)=u_{\varepsilon}+\hat{u}$ and $u_{t}(\cdot, 0)=\hat{v}$.

Theorem 4.3 For $\varepsilon>0$ but sufficiently small, the travelling wave solution $u_{\varepsilon}$ of (1.1) is asymptotically stable.

Remark 4.4 A linearized stability result has also been obtained by Maginu in [16]. In this paper the point spectrum near 0 is determined in the case $\beta=0$. In addition the stability of periodic wave trains is determined, which we do not touch upon at all in this paper.

\subsection{The Evans function for $\lambda$ near 0}

The existence of the travelling wave front solution in the perturbed system, gives rise to an eigenvalue $\lambda=0$. Indeed, the front solution $u_{\varepsilon}(\xi)$ satisfies

$$
-\left(u_{\varepsilon}\right)_{\xi \xi}+\sin u_{\varepsilon}=\varepsilon\left(\gamma+\frac{\alpha c_{\varepsilon}}{\sqrt{1-c_{\varepsilon}^{2}}}\left(u_{\varepsilon}\right)_{\xi}\right) .
$$

Differentiating this equation with respect to $\xi$ gives that $v(\xi, t)=\left(u_{\varepsilon}\right)_{\xi}$ is a solution of the linearised problem (3.1) with $\beta=0$. We define $\widetilde{V}^{\varepsilon}(\xi)=\frac{1}{2}\left(u_{\varepsilon},\left(u_{\varepsilon}\right)_{\xi}\right)^{T}$ and $U^{+}(\xi, 0, \varepsilon)=$ $C_{\varepsilon}^{+} \widetilde{V}_{\xi}^{\varepsilon}(\xi), U^{-}(\xi, 0, \varepsilon)=C_{\varepsilon}^{-} \widetilde{V}_{\xi}^{\varepsilon}(\xi)$, where $C_{\varepsilon}^{ \pm}$are such that the normalisation (3.9) is satisfied. From the previous section, it follows that $C_{0}^{ \pm}=\frac{1}{2}$. Note that the definition of $\widetilde{V}_{\xi}^{\varepsilon}(\xi)$ agrees 
with the unperturbed limit, i.e. the solution of the unperturbed linearised system (see (3.10)). Since $U^{+}$and $U^{-}$are parallel, it follows immediately that $D(0, \varepsilon)=0$. We can also determine the behaviour of the Evans function near $\lambda=0$.

Lemma 4.5 The Evans function satisfies $D(0, \varepsilon)=0$ and $\frac{\partial}{\partial \lambda} D(0, \varepsilon)=\frac{\varepsilon \alpha}{2\left(1-c_{0}^{2}\right)}+\mathcal{O}\left(\varepsilon^{2}\right)$.

Corollary 4.6 For $\lambda$ and $\varepsilon$ near 0, the Evans function is given by

$$
D(\lambda, \varepsilon)=\frac{\alpha \varepsilon \lambda}{2\left(1-c_{0}^{2}\right)}+\frac{\lambda^{2}}{2\left(1-c_{0}^{2}\right)}+\mathcal{O}\left(\lambda^{3}+\lambda^{2} \varepsilon+\lambda \varepsilon^{2}\right) .
$$

Hence the Evans function is positive if $\alpha>0$ and $\lambda>0$ and the eigenvalue bifurcation from $\lambda=0$ goes into the left half plane. To be explicit, for $\varepsilon$ small this eigenvalue is $\lambda=-\varepsilon \alpha+\mathcal{O}\left(\varepsilon^{2}\right)$.

Proof of Lemma 4.5 We calculate the derivative of the Evans function with respect to $\lambda$ at $\lambda=0$.

$$
\begin{aligned}
\frac{\partial}{\partial \lambda} D(0, \varepsilon)= & e^{-\int_{0}^{\xi} \operatorname{tr}(\mathbf{A}(s, 0, \varepsilon)) d s} \\
& \left(\frac{\partial}{\partial \lambda} U^{+}(\xi, 0, \varepsilon) \wedge C_{\varepsilon}^{-} \widetilde{V}_{\xi}^{\varepsilon}(\xi)+C_{\varepsilon}^{+} \widetilde{V}_{\xi}^{\varepsilon}(\xi) \wedge \frac{\partial}{\partial \lambda} U^{-}(\xi, 0, \varepsilon)\right) \\
= & W^{+}(\xi, \varepsilon)-W^{-}(\xi, \varepsilon),
\end{aligned}
$$

where

$$
W^{ \pm}(\xi, \varepsilon)=C_{\varepsilon}^{\mp} e^{-\int_{0}^{\xi} \operatorname{tr}(\mathbf{A}(s, 0, \varepsilon)) d s} \frac{\partial}{\partial \lambda} U^{ \pm}(\xi, 0, \varepsilon) \wedge \widetilde{V}_{\xi}^{\varepsilon}(\xi) .
$$

Hence we have to analyse the derivative of $U^{ \pm}$with respect to $\lambda$. By differentiating (3.3) with respect to $\lambda$ and setting $\lambda=0$, we see that $\frac{\partial}{\partial \lambda} U^{ \pm}(\xi, 0, \varepsilon)$ satisfies

$$
\left(\frac{\partial}{\partial \lambda} U^{ \pm}(\xi, 0, \varepsilon)\right)_{\xi}=\mathbf{A}(\xi, 0, \varepsilon) \frac{\partial}{\partial \lambda} U^{ \pm}(\xi, 0, \varepsilon)+\mathbf{M}(\varepsilon) C_{\varepsilon}^{ \pm} \widetilde{V}_{\xi}^{\varepsilon}(\xi)
$$

where

$$
\mathbf{M}(\varepsilon)=\frac{\partial}{\partial \lambda} \mathbf{A}(\xi, 0, \varepsilon)=\left(\begin{array}{cc}
0 & 0 \\
\varepsilon \alpha & \frac{-2 c_{\varepsilon}}{\sqrt{1-c_{\varepsilon}^{2}}}
\end{array}\right)
$$

Thus, for the $\xi$-derivative of $W^{ \pm}(\xi, \varepsilon)$ we get

$$
\begin{aligned}
D_{\xi} W^{ \pm}(\xi, \varepsilon)= & -\operatorname{tr}(\mathbf{A}(\xi, 0, \varepsilon)) W^{ \pm}(\xi, \varepsilon) \\
& +C_{\varepsilon}^{\mp} e^{-\int_{0}^{\xi} \operatorname{tr}(\mathbf{A}(s, 0, \varepsilon)) d s} \mathbf{A}(\xi, 0, \varepsilon) \frac{\partial}{\partial \lambda} U^{ \pm}(\xi, 0, \varepsilon) \wedge \widetilde{V}_{\xi}^{\varepsilon}(\xi) \\
& +C_{\varepsilon}^{\mp} e^{-\int_{0}^{\xi} \operatorname{tr}(\mathbf{A}(s, 0, \varepsilon)) d s} \mathbf{M}(\varepsilon) C_{\varepsilon}^{ \pm} \widetilde{V}_{\xi}^{\varepsilon}(\xi) \wedge \widetilde{V}_{\xi}^{\varepsilon}(\xi) \\
& +C_{\varepsilon}^{\mp} e^{-\int_{0}^{\xi} \operatorname{tr}(\mathbf{A}(s, 0, \varepsilon)) d s} \frac{\partial}{\partial \lambda} U^{ \pm}(\xi, \varepsilon) \wedge \mathbf{A}(\xi, 0, \varepsilon) \widetilde{V}_{\xi}^{\varepsilon}(\xi, c) \\
= & C_{\varepsilon}^{\mp} C_{\varepsilon}^{ \pm} e^{-\int_{0}^{\xi} \operatorname{tr}(\mathbf{A}(s, 0, \varepsilon)) d s} \mathbf{M}(\varepsilon) \widetilde{V}_{\xi}^{\varepsilon}(\xi) \wedge \widetilde{V}_{\xi}^{\varepsilon}(\xi) .
\end{aligned}
$$

The last equality follows from the fact that for any two vectors $U_{1}$ and $U_{2}$ and any matrix $\mathbf{B}$ we have $\mathbf{B} U_{1} \wedge U_{2}+U_{1} \wedge \mathbf{B} U_{2}=\operatorname{tr}(\mathbf{B}) U_{1} \wedge U_{2}$. Using that $\lim _{\xi \rightarrow \pm \infty} W^{ \pm}(\xi, \varepsilon)=0$, we conclude that

$$
\begin{aligned}
\frac{\partial}{\partial \lambda} D(0, \varepsilon) & =W^{+}(\xi, \varepsilon)-W^{-}(\xi, \varepsilon) \\
& =-C_{\varepsilon}^{\mp} C_{\varepsilon}^{ \pm} \int_{-\infty}^{\infty} e^{-\int_{0}^{\xi} \operatorname{tr}(\mathbf{A}(s, 0, \varepsilon)) d s} \mathbf{M}(\varepsilon) \tilde{V}_{\xi}^{\varepsilon}(\xi) \wedge \tilde{V}_{\xi}^{\varepsilon}(\xi) d \xi
\end{aligned}
$$


where $\operatorname{tr}(\mathbf{A}(\xi, 0, \varepsilon))=-\frac{\varepsilon c_{\varepsilon} \alpha}{\sqrt{1-c_{\varepsilon}^{2}}}$. Using the definition of $\mathbf{M}$ (4.1) we have

$$
\mathbf{M} \widetilde{V}_{\xi}^{\varepsilon}(\xi) \wedge \widetilde{V}_{\xi}^{\varepsilon}(\xi)=\frac{1}{4}\left(-\varepsilon \alpha\left(\left(u_{\varepsilon}\right)_{\xi}\right)^{2}+\frac{c_{\varepsilon}}{\sqrt{1-c_{\varepsilon}^{2}}} \frac{\partial}{\partial \xi}\left(\left(u_{\varepsilon}\right)_{\xi}\right)^{2}\right)
$$

Hence

$$
\frac{\partial}{\partial \lambda} D(0, \varepsilon)=\frac{\alpha \varepsilon C_{\varepsilon}^{\mp} C_{\varepsilon}^{ \pm}}{4\left(1-c_{\varepsilon}^{2}\right)} \int_{-\infty}^{\infty} e^{\frac{\varepsilon c_{\varepsilon} \alpha}{\sqrt{1-c_{\varepsilon}^{2}}} \xi}\left(\left(u_{\varepsilon}\right)_{\xi}\right)^{2} d \xi=\frac{\alpha \varepsilon}{2\left(1-c_{0}^{2}\right)}+\mathcal{O}\left(\varepsilon^{2}\right),
$$

since $\left(u_{0}\right)_{\xi}(\xi)=2 \operatorname{sech} \xi$ and $C_{\varepsilon}^{ \pm}=\frac{1}{2}+\mathcal{O}(\varepsilon)$. Thus, we conclude that for $\lambda$ small,

$$
D(\lambda, \varepsilon)=\frac{\alpha \varepsilon \lambda}{2\left(1-c_{0}^{2}\right)}+\frac{\lambda^{2}}{2\left(1-c_{0}^{2}\right)}+\mathcal{O}\left(\lambda^{2} \varepsilon+\lambda^{3} \varepsilon+\lambda^{4}\right)
$$

(Lemma 3.4). The expression for the bifurcating eigenvalue follows by setting $D(\lambda, \varepsilon)=0$.

\subsection{The Evans function for $\lambda$ near $\pm \mathrm{i} \omega_{0}$ and $\varepsilon$ near zero}

Instability might again occur through eigenvalues that bifurcate from the endpoints of the continuous spectrum, i.e., from $\lambda= \pm \mathrm{i} \omega_{0}$. As seen before, $\lambda$ is the only non-real element in the linearised problem, hence the Evans function satisfies $D(\bar{\lambda}, \varepsilon)=\overline{D(\lambda, \varepsilon)}$. So we can restrict the analysis to the case $\lambda=\mathrm{i} \omega_{0}$.

The analysis follows the ideas of the analysis of the bifurcation from the endpoint of the continuous spectrum in [11] and it has to be done in a rather subtle way. Firstly an $\varepsilon$-dependent near-identity coordinate transformation has to be made from $\lambda$ to $\sigma$ such that the end of the continuous spectrum occurs at a fixed value of the new coordinate $\sigma$ for all $\varepsilon$, namely $\sigma=\mathrm{i} \omega_{0}$. Furthermore, the Evans function is not analytic anymore for $\sigma$ near $\mathrm{i} \omega_{0}$, but it is smooth in $\sqrt{\sigma-i \omega_{0}}$, as will be seen later. An extra complication compared to the analysis in [11] is that second derivative of the Evans function has to be determined, since the first derivative vanishes.

Recall the characteristic polynomial (3.5). A double eigenvalue occurs at

$$
\lambda=\mathrm{i} \omega_{0}+\widetilde{\lambda}(\varepsilon)=\mathrm{i} w_{\varepsilon}-\frac{\varepsilon \alpha}{2},
$$

where

$$
\widetilde{\lambda}(\varepsilon)=\mathrm{i} \omega_{\varepsilon}-\mathrm{i} \omega_{0}-\frac{\varepsilon \alpha}{2}=\mathrm{i} \sqrt{1-c_{\varepsilon}^{2}} \sqrt{\sqrt{1-\varepsilon^{2} \gamma^{2}}-\frac{\alpha^{2} \varepsilon^{2}}{4}}-\mathrm{i} \sqrt{1-c_{0}^{2}}-\frac{\varepsilon \alpha}{2} .
$$

This double eigenvalue occurs at the end of the continuous spectrum. Note that $\widetilde{\lambda}(\varepsilon)+\frac{\varepsilon \alpha}{2}$ is purely imaginary and a function of $\varepsilon^{2}$, since $c_{-\varepsilon}=c_{\varepsilon}$ (Lemma 2.3).

Define the coordinate transformation $\sigma=\lambda-\widetilde{\lambda}(\varepsilon)$ and the matrix

$$
\widehat{\mathbf{A}}(\xi, \sigma, \varepsilon)=\mathbf{A}(\xi, \lambda, \varepsilon)=\left(\begin{array}{cc}
0 & 1 \\
\left(\sigma+\mathrm{i}\left(\omega_{\varepsilon}-\omega_{0}\right)\right)^{2}-\frac{\varepsilon^{2} \alpha^{2}}{4}+\cos \left(u_{\varepsilon}(\xi)\right) & -\frac{2 c_{\varepsilon}\left(\sigma+\mathrm{i}\left(\omega_{\varepsilon}-\omega_{0}\right)\right)}{\sqrt{1-c_{\varepsilon}^{2}}}
\end{array}\right) .
$$

Then $\widehat{\mathbf{A}}(\xi, \sigma, \varepsilon)=\widehat{\mathbf{A}}(-\xi, \sigma,-\varepsilon)$ since $u_{-\varepsilon}(-\xi)=2 \pi-u_{\varepsilon}(\xi)$ and $c_{-\varepsilon}=c_{\varepsilon}$ (see Lemma 2.3). Also, the asymptotic matrix

$$
\widehat{\mathbf{A}}_{0}(\sigma, \varepsilon)=\widehat{\mathbf{A}}( \pm \infty, \sigma, \varepsilon)=\mathbf{A}_{0}(\lambda, \varepsilon)
$$


has a purely imaginary eigenvalue

$$
\mu_{d}\left(\varepsilon^{2}\right)=-\mathrm{i} c_{\varepsilon} \sqrt{\sqrt{1-\varepsilon^{2} \gamma^{2}}-\frac{\alpha^{2} \varepsilon^{2}}{4}}
$$

at $\sigma=\mathrm{i} \omega_{0}$ of algebraic multiplicity 2 and geometric multiplicity 1 with eigenvector

$$
\eta_{d}\left(\varepsilon^{2}\right)=\left(\begin{array}{c}
1 \\
\mu_{d}\left(\varepsilon^{2}\right)
\end{array}\right)
$$

We also define the solution vectors $\widehat{U}^{ \pm}(\xi, \sigma, \varepsilon)=U^{ \pm}(\xi, \sigma+\widetilde{\lambda}(\varepsilon), \varepsilon)$. Using the symmetries in the equation, we can derive a relation between $\widehat{U}^{+}$and $\widehat{U}^{-}$. First we note that the matrix

$$
\widehat{\mathbf{A}}\left(\xi, \mathrm{i} \omega_{0}, \varepsilon\right)=\left(\begin{array}{cc}
0 & 1 \\
\frac{1-c_{\varepsilon}^{2}}{c_{\varepsilon}^{2}} \mu_{d}^{2}\left(\varepsilon^{2}\right)-\frac{\varepsilon^{2} \alpha^{2}}{4}+\cos \left(u_{\varepsilon}(\xi)\right) & 2 \mu_{d}\left(\varepsilon^{2}\right)
\end{array}\right) .
$$

Since $u_{-\varepsilon}(-\xi)=2 \pi-u_{\varepsilon}(\xi), c_{-\varepsilon}=c_{\varepsilon}$ and $\mu_{d}\left(\varepsilon^{2}\right)$ is purely imaginary, this implies that

$$
\widehat{\mathbf{A}}\left(-\xi, \mathrm{i} \omega_{0},-\varepsilon\right)=\widehat{\mathbf{A}}\left(\xi, \mathrm{i} \omega_{0}, \varepsilon\right) \text { and } \overline{\widehat{\mathbf{A}}\left(\xi, \mathrm{i} \omega_{0}, \varepsilon\right)}=-S \widehat{\mathbf{A}}\left(\xi, \mathrm{i} \omega_{0}, \varepsilon\right) S,
$$

where $S=\operatorname{diag}(1,-1)$ as before. From these properties, it follows that

$$
\frac{\partial}{\partial \xi}\left[\widehat{S \widehat{U}^{ \pm}\left(-\xi, \mathrm{i} \omega_{0},-\varepsilon\right)}\right]=\widehat{\mathbf{A}}\left(\xi, \mathrm{i} \omega_{0}, \varepsilon\right) \overline{S \widehat{U}^{ \pm}\left(-\xi, \mathrm{i} \omega_{0}, \varepsilon\right)}
$$

Furthermore,

$$
\lim _{\xi \rightarrow \mp \infty} e^{-\mu_{d}\left(\varepsilon^{2}\right) \xi} \overline{S \widehat{U}^{ \pm}\left(-\xi, \mathrm{i} \omega_{0},-\varepsilon\right)}=\overline{S \mu_{d}\left(\varepsilon^{2}\right)}=\mu_{d}\left(\varepsilon^{2}\right) .
$$

So we can conclude that

$$
\overline{S \widehat{U}^{ \pm}\left(-\xi, \mathrm{i} \omega_{0},-\varepsilon\right)}=\widehat{U}^{\mp}(\xi, \varepsilon) .
$$

Finally we define the Evans function $\widehat{D}(\sigma, \varepsilon)=D(\lambda, \varepsilon)$. Clearly the function $\widehat{D}(\sigma, \varepsilon)$ is not analytic at $\sigma=\mathrm{i} \omega_{0}$, because of the double eigenvalue. However, at $\sigma=\mathrm{i} \omega_{0}$, it is still smooth in $\varepsilon$.

The first result we obtain is

Lemma 4.7 $\widehat{D}\left(\mathrm{i} \omega_{0}, 0\right)=0=\frac{\partial}{\partial \varepsilon} \widehat{D}\left(\mathrm{i} \omega_{0}, 0\right)$.

The fact that the first derivative of the Evans function with respect to $\varepsilon$ vanishes at the point $\left(\mathrm{i} \omega_{0}, 0\right)$ should not come as a surprise, since at $\xi= \pm \infty$, the matrix $\widehat{\mathbf{A}}$ depends on $\varepsilon^{2}$.

This result is sufficient to establish the stability of the wave. Even if there are eigenvalues bifurcating from the continuous spectrum, they will be so close (at a distance smaller than $\left.\mathcal{O}\left(\varepsilon^{2}\right)\right)$ to the continuous spectrum - that is $\mathcal{O}(\varepsilon)$ away from the imaginary axis - that they cannot cause instability.

Nevertheless, we need to go one step further to find out whether or not 'new' eigenvalues appear:

Lemma 4.8 For all $\varepsilon$ small $\widehat{D}\left(\mathrm{i} \omega_{0}, \varepsilon\right) \approx-1.1571 \gamma^{2} \varepsilon^{2}+o\left(\varepsilon^{2}\right)$. No eigenvalues bifurcate from the continuous spectrum. 
In this Lemma, the value of the second order term $\left(\mathcal{O}\left(\varepsilon^{2}\right)\right)$ of the Evans function is determined by numerical integration of explicitly determined (but computationally rather complicated) integrals. The proof of this Lemma is given in Appendix A.

Proof of Lemma 4.7 It follows from Lemma 3.4 and section 3.2 that $\widehat{D}\left(\mathrm{i} \omega_{0}, 0\right)=0$. The definition of the Evans function implies that

$$
\begin{aligned}
\frac{\partial}{\partial \varepsilon} \widehat{D}\left(\mathrm{i} \omega_{0}, 0\right) & =e^{-2 \mu_{d}(0) \xi}\left(\frac{\partial}{\partial \varepsilon} \widehat{U}^{+} \wedge \widehat{U}^{-}+\widehat{U}^{+} \wedge \frac{\partial}{\partial \varepsilon} \widehat{U}^{-}\right)\left(\xi, \mathrm{i} \omega_{0}, 0\right) \\
& =\left(W^{+}-W^{-}\right)\left(\xi, \mathrm{i} \omega_{0}, 0\right),
\end{aligned}
$$

where

$$
W^{ \pm}\left(\xi, \mathrm{i} \omega_{0}, \varepsilon\right)=e^{-2 \mu_{d}\left(\varepsilon^{2}\right) \xi}\left(\frac{\partial}{\partial \varepsilon} \widehat{U}^{ \pm} \wedge \widehat{U}^{\mp}\right)\left(\xi, \mathrm{i} \omega_{0}, \varepsilon\right) .
$$

We compute $\frac{\partial}{\partial \xi} W^{ \pm}\left(\xi, \mathrm{i} \omega_{0}, 0\right)$,

$$
\begin{aligned}
\frac{\partial}{\partial \xi} W^{ \pm}\left(\xi, \mathrm{i} \omega_{0}, 0\right)= & -2 \mu_{d}(0) W^{ \pm}\left(\xi, \mathrm{i} \omega_{0}, 0\right)+ \\
& e^{-2 \mu_{d}(0) \xi}\left(\frac{\partial^{2} \widehat{U}^{ \pm}}{\partial \xi \partial \varepsilon} \wedge \widehat{U}^{\mp}+\frac{\partial \widehat{U}^{ \pm}}{\partial \varepsilon} \wedge \frac{\partial \widehat{U}^{\mp}}{\partial \xi}\right)\left(\xi, \mathrm{i} \omega_{0}, 0\right) .
\end{aligned}
$$

As both $\widehat{U}^{ \pm}$are solutions of the linear equation $U_{\xi}=\widehat{\mathbf{A}} U$, we find by differentiation with respect to $\varepsilon$ that

$$
\frac{\partial^{2} \widehat{U}^{ \pm}}{\partial \xi \partial \varepsilon}=\frac{\partial \widehat{\mathbf{A}}}{\partial \varepsilon} \widehat{U}^{ \pm}+\widehat{\mathbf{A}} \frac{\partial \widehat{U}^{ \pm}}{\partial \varepsilon} .
$$

Hence, we conclude

$$
\begin{aligned}
\frac{\partial}{\partial \xi} W^{ \pm}\left(\xi, \mathrm{i} \omega_{0}, 0\right)=-2 \mu_{d}(0) W^{ \pm}\left(\xi, \mathrm{i} \omega_{0}, 0\right)+ \\
\quad e^{-2 \mu_{d}(0) \xi}\left(\frac{\partial \widehat{\mathbf{A}}}{\partial \varepsilon} \widehat{U}^{ \pm} \wedge \widehat{U}^{\mp}+\widehat{\mathbf{A}} \frac{\partial \widehat{U}^{ \pm}}{\partial \varepsilon} \wedge \widehat{U}^{\mp}+\frac{\partial \widehat{U}^{ \pm}}{\partial \varepsilon} \wedge \widehat{\mathbf{A}} \widehat{U}^{\mp}\right)\left(\xi, \mathrm{i} \omega_{0}, 0\right) \\
=-2 \mu_{d}(0) W^{ \pm}\left(\xi, \mathrm{i} \omega_{0}, 0\right)+e^{-2 \mu_{d}(0) \xi} \operatorname{trace}\left(\widehat{\mathbf{A}}\left(\xi, \mathrm{i} \omega_{0}, 0\right)\right) \frac{\partial \widehat{U}^{ \pm}}{\partial \varepsilon} \wedge \widehat{U}^{\mp}\left(\xi, \mathrm{i} \omega_{0}, 0\right) \\
\quad+e^{-2 \mu_{d}(0) \xi} \frac{\partial \widehat{\mathbf{A}}}{\partial \varepsilon} \widehat{U}^{ \pm} \wedge \widehat{U}^{\mp}\left(\xi, \mathrm{i} \omega_{0}, 0\right) \\
=e^{-2 \mu_{d}(0) \xi} \frac{\partial \widehat{\mathbf{A}}}{\partial \varepsilon} \widehat{U}^{ \pm} \wedge \widehat{U}^{\mp}\left(\xi, \mathrm{i} \omega_{0}, 0\right) .
\end{aligned}
$$

Integrating $\frac{\partial}{\partial \xi} W^{+}\left(\xi, \mathrm{i} \omega_{0}, 0\right)$ from 0 to $\infty$ and $\frac{\partial}{\partial \xi} W^{-}\left(\xi, \mathrm{i} \omega_{0}, 0\right)$ from 0 to $-\infty$ and observing that

$$
\widehat{U}^{ \pm}\left(\xi, \mathrm{i} \omega_{0}, 0\right)= \pm e^{-\mathrm{i} c_{0} \xi}\left(\tanh \xi,-\mathrm{i} c_{0} \tanh \xi+\operatorname{sech}^{2} \xi\right) \stackrel{\text { def }}{=} \pm \widehat{U}(\xi)
$$

(see section 3.1), we arrive at the following expression:

$$
\begin{aligned}
\frac{\partial}{\partial \varepsilon} \widehat{D}\left(\mathrm{i} \omega_{0}, 0\right)= & -\int_{\infty}^{-\infty} e^{-2 \mu_{d}(0) \xi}\left(\frac{\partial \widehat{\mathbf{A}}}{\partial \varepsilon}\left(\xi, \mathrm{i} \omega_{0}, 0\right) \widehat{U}(\xi) \wedge \widehat{U}(\xi)\right) d \xi \\
& +W^{+}\left(\infty, \mathrm{i} \omega_{0}, 0\right)-W^{-}\left(-\infty, \mathrm{i} \omega_{0}, 0\right)
\end{aligned}
$$


where

$$
W^{ \pm}\left( \pm \infty, \mathrm{i} \omega_{0}, 0\right)=\lim _{\xi \rightarrow \pm \infty} e^{-2 \mu_{d}(0) \xi}\left(\frac{\partial \widehat{U}^{ \pm}}{\partial \varepsilon} \wedge \widehat{U}^{\mp}\right)\left(\xi, \mathrm{i} \omega_{0}, 0\right) .
$$

By definition, $\lim _{\xi \rightarrow \pm \infty} e^{-\mu_{d}\left(\varepsilon^{2}\right) \xi} \widehat{U}^{ \pm}\left(\xi, \mathrm{i} \omega_{0}, \varepsilon\right)=\eta_{d}\left(\varepsilon^{2}\right)$. Differentiating this relation with respect to $\varepsilon$ and substituting $\varepsilon=0$, gives $\left.\lim _{\xi \rightarrow \pm \infty} e^{-i c_{0} \xi} \frac{\partial}{\partial \varepsilon}\right|_{\varepsilon=0} \widehat{U}^{ \pm}\left(\xi, \mathrm{i} \omega_{0}, \varepsilon\right)=(0,0)$. Hence $W^{ \pm}\left( \pm \infty, \mathrm{i} \omega_{0}, 0\right)=0$ and

$$
\frac{\partial}{\partial \varepsilon} \widehat{D}\left(\mathrm{i} \omega_{0}, 0\right)=-\int_{\infty}^{-\infty} e^{-2 \mathrm{i} c_{0} \xi}\left(\frac{\partial \widehat{\mathbf{A}}}{\partial \varepsilon}\left(\xi, \mathrm{i} \omega_{0}, 0\right) \widehat{U}(\xi) \wedge \widehat{U}(\xi)\right) d \xi
$$

Since

$$
\frac{\partial}{\partial \varepsilon} \widehat{A}\left(\xi, \mathrm{i} \omega_{0}, 0\right)=\left(\begin{array}{cc}
0 & 0 \\
-\sin \left(u_{0}(\xi)\right) u_{1}(\xi) & 0
\end{array}\right),
$$

with $u_{1}(\xi)=\left.\frac{\partial u_{\varepsilon}(\xi)}{\partial \varepsilon}\right|_{\varepsilon=0}$, this leads to the expression

$$
\frac{\partial}{\partial \varepsilon} \widehat{D}\left(\mathrm{i} \omega_{0}, 0\right)=-\int_{\infty}^{-\infty} \sin \left(u_{0}(\xi)\right) u_{1}(\xi) \tanh ^{2}(\xi) d \xi=0
$$

as the integrand is odd, see Lemma 2.3.

\section{$5 \quad$ Stability analysis for a small surface resistance}

In section 3.2 we have determined the "unperturbed" Evans function for the singular case $(\beta \neq$ 0 ) and we have seen that a possible bifurcation can occur at $\lambda=0$ or $\lambda= \pm \mathrm{i} \omega_{0}$. A careful analysis of the singularly perturbed Evans function $D^{\beta}(\lambda, \varepsilon)$ yields that the perturbations cannot destabilise the stable unperturbed wave (under the natural, physical conditions, $\alpha, \beta>$ $0)$.

Theorem 5.1 Let $\alpha, \beta>0, \gamma \in \mathbb{R}$ and $\varepsilon>0$ be small enough. Then, the perturbed travelling wave solution $u_{\varepsilon}(\xi)$ of (1.1) is spectrally stable. The linearised stability problem has two real eigenvalues,

$$
\lambda_{1}=0 \text { and } \lambda_{2}(\varepsilon)=-\varepsilon\left(\alpha+\frac{\beta\left(1+2 c_{0}^{2}\right)}{3\left(1-c_{0}^{2}\right)}\right)+\mathcal{O}\left(\varepsilon^{2}\right)<0,
$$

and no eigenvalues bifurcating out of the continuous spectrum.

Remark 5.2 Unlike in the regular problem (i.e. $\beta=0$ ), the corresponding result on the nonlinear stability of $u_{\varepsilon}(\xi)$ is not a (relatively) straightforward extension of similar statements in the literature. Here, we do not go into the details of the nonlinear stability of $u_{\varepsilon}(\xi)$ in the singular problem. However, we conjecture that a result similar to that of Theorem 4.3 can also be obtained for the case $\beta \neq 0$.

The general idea behind the proof of this Theorem is like that of the previous section. However, we shall find that the singularly perturbed nature of the $\beta \neq 0$ problem indeed makes the analysis more subtle.

Before we can study the Evans function $D^{\beta}(\lambda, \varepsilon)$, we need to determine a more accurate approximation of the fast solution $U^{f}(\xi, \lambda, \varepsilon)$. 


\subsection{The fast solution}

The eigenvector with the eigenvalue $\mu_{3}$ is $\eta_{3}=\left(\frac{1}{\mu_{3}^{2}}, \frac{1}{\mu_{3}}, 1\right)$ (3.18), hence the eigenvector corresponding to the fast eigenvalue $\mu_{3}$ is

$$
\eta_{3}(\lambda, \varepsilon)=(0,0,1)+\varepsilon\left(0, \frac{\beta c_{0}}{\left(\sqrt{1-c_{0}^{2}}\right)^{3}}, 0\right)+\varepsilon^{2}\left(\frac{\beta^{2} c_{0}^{2}}{\left(1-c_{0}^{2}\right)^{3}},-\frac{\beta^{2} c_{0}\left(1+c_{0}^{2}\right)}{\left(\sqrt{1-c^{2}}\right)^{7}} \lambda, 0\right)+\mathcal{O}\left(\varepsilon^{3}\right)
$$

(see (3.16)). Note that we have used here that $c_{\varepsilon}=c_{0}+\mathcal{O}\left(\varepsilon^{2}\right)$ (Lemma 2.3). We expect the fast solution $U^{f}(\xi, \lambda, \varepsilon)$ to be $\mathcal{O}(\varepsilon)$ close to $\eta_{3}$, therefore we decompose $U^{f}$ into

$$
U^{f}(\xi, \lambda, \varepsilon)=\left[\eta_{3}(\lambda, \varepsilon)+\varepsilon Z(\xi, \lambda, \varepsilon)\right] e^{\mu_{3}(\lambda, \varepsilon) \xi},
$$

where $Z(\xi)=\left(z_{1}(\xi), z_{2}(\xi), z_{3}(\xi)\right)$ must go to zero as $\xi \rightarrow-\operatorname{sgn}\left(\beta c_{\varepsilon}\right) \infty$ and be bounded at the other end. Substitution into the linearised system gives

$$
\begin{aligned}
& \varepsilon\left(z_{1}\right)_{\xi}+\varepsilon \mu_{3} z_{1}=\varepsilon z_{2} \\
& \varepsilon\left(z_{2}\right)_{\xi}+\varepsilon \mu_{3} z_{2}=\varepsilon z_{3} \\
& \varepsilon\left(z_{3}\right)_{\xi}+\varepsilon \mu_{3} z_{3}=-\frac{\left(\lambda^{2}+\cos u_{\varepsilon}\right)\left(\sqrt{1-c_{0}^{2}}\right)^{3} z_{1}}{\beta c_{0}}+\frac{2 \lambda\left(1-c_{0}^{2}\right) z_{2}}{\beta}+\frac{\left(\sqrt{1-c_{0}^{2}}\right)^{3} z_{3}}{\beta c_{0}} \\
& +\varepsilon\left(-\frac{\alpha \lambda}{\beta c_{0}}{\sqrt{1-c_{0}^{2}}}^{3} z_{1}+\frac{\alpha\left(1-c_{0}^{2}\right)}{\beta} z_{2}+\frac{\lambda \sqrt{1-c_{0}^{2}}}{c_{0}} z_{3}+\left(1-\cos u_{0}\right) \frac{\beta c_{0}}{\sqrt{1-c_{0}^{2}}}\right) \\
& +\mathcal{O}\left(\varepsilon^{2}\right) \text {. }
\end{aligned}
$$

Since $\varepsilon \mu_{3}(\lambda, \varepsilon)=\frac{\left(\sqrt{1-c_{0}^{2}}\right)^{3}}{\beta c_{0}}+\frac{\varepsilon \lambda\left(1+c_{0}^{2}\right)}{c_{0} \sqrt{1-c_{0}^{2}}}+\mathcal{O}\left(\varepsilon^{2}\right)(3.16)$, we immediately find that

$$
z_{2}=\mathcal{O}(\varepsilon), \quad z_{1}=\mathcal{O}\left(\varepsilon^{2}\right) .
$$

Substitution of $z_{1}$ and $z_{2}$ into the $z_{3}$-equation of (5.1) yields $\varepsilon\left(z_{3}\right)_{\xi}=\mathcal{O}(\varepsilon)$ which implies that we have to go one order higher in the expansion to obtain $z_{3}(\xi)$. We find

$$
\left(z_{3}\right)_{\xi}=\frac{\beta c_{0}}{\left(\sqrt{1-c_{0}^{2}}\right)^{3}}\left(1-\cos \left(u_{0}(\xi)\right)\right)+\mathcal{O}(\varepsilon) .
$$

Of course $\left(u_{0}\right)_{\xi \xi}-\sin u_{0}=0$, therefore $\cos u_{0}=\left(u_{0}\right)_{\xi \xi \xi} /\left(u_{0}\right)_{\xi}=2 \tanh ^{2} \xi-1$. Also $Z(\xi) \rightarrow 0$ for $\xi \rightarrow-\operatorname{sgn}\left(\beta c_{\varepsilon}\right) \infty$, thus, at leading order,

$$
z_{3}(\xi)=\frac{\beta c_{0}}{\left(\sqrt{1-c_{0}^{2}}\right)^{3}} \int_{-\operatorname{sgn}\left(\beta c_{0}\right) \infty}^{\xi}\left(1-\cos \left(u_{0}(\xi)\right)\right) d \xi=2 \frac{\beta c_{0}}{\left(\sqrt{1-c_{0}^{2}}\right)^{3}}\left(\tanh (\xi)+\frac{\beta c_{0}}{\mid \beta c_{0}}\right) .
$$

Inserting this into (5.1) yields the next order approximation of $z_{2}$ :

$$
z_{2}(\xi)=2 \varepsilon \frac{\beta^{2} c_{0}^{2}}{\left(1-c_{0}^{2}\right)^{3}}\left(\tanh (\xi)+\frac{\beta c_{0}}{\left|\beta c_{0}\right|}\right)+\mathcal{O}\left(\varepsilon^{2}\right) .
$$

So, altogether we have

$$
U^{f}(\xi, \lambda, \varepsilon)=\left(\begin{array}{c}
\varepsilon^{2} \frac{\beta^{2} c_{0}^{2}}{\left(1-c_{0}^{2}\right)^{3}}+\mathcal{O}\left(\varepsilon^{3}\right) \\
\varepsilon \frac{\beta c_{0}}{\left(\sqrt{1-c_{0}^{2}}\right)^{3}}-\varepsilon^{2}\left(2 \frac{\beta^{2} c_{0}^{2}}{\left(1-c_{0}^{2}\right)^{3}}\left(\tanh (\xi)+\frac{\beta c_{0}}{\beta c_{0} \mid}\right)-\frac{\beta^{2} c_{0}\left(1+c_{0}^{2}\right)}{\left(\sqrt{1-c^{2}}\right)^{7}} \lambda\right)+\mathcal{O}\left(\varepsilon^{3}\right) \\
1+2 \varepsilon \frac{\beta c_{0}}{\left(\sqrt{1-c_{0}^{2}}\right)^{3}}\left(\tanh (\xi)+\frac{\beta c_{0}}{\beta c_{0} \mid}\right)+\mathcal{O}\left(\varepsilon^{2}\right)
\end{array}\right) e^{\mu_{3}(\lambda, \varepsilon) \xi}
$$


This approximation is uniform in $\xi$.

\subsection{The Evans function for $\lambda$ near 0}

The existence of the travelling wave front solution in the perturbed system of course again gives rise to an eigenvalue $\lambda=0: v(\xi, t)=\left(u_{\varepsilon}\right)_{\xi}$ is a solution of the linearised problem (3.1) at $\lambda=0$. In this section we derive an expression for the derivative of $D^{\beta}(\lambda, \varepsilon)$ with respect to $\lambda$ at $\lambda=0$.

Lemma 5.3 $D^{\beta}(\lambda, \varepsilon)$ satisfies $D^{\beta}(0, \varepsilon)=0$ and $\frac{\partial}{\partial \lambda} D^{\beta}(0, \varepsilon)=\frac{3 \alpha\left(1-c_{0}^{2}\right)+\left(1+2 c_{0}^{2}\right) \beta}{6\left(1-c_{0}^{2}\right)^{4}} \varepsilon+\mathcal{O}\left(\varepsilon^{2}\right)$.

This immediately implies

Corollary $5.4 D^{\beta}(\lambda, \varepsilon)$ satisfies

$$
D^{\beta}(\lambda, \varepsilon)=\frac{3 \alpha\left(1-c_{0}^{2}\right)+\left(1+2 c_{0}^{2}\right) \beta}{6\left(1-c_{0}^{2}\right)^{4}} \varepsilon \lambda+\frac{1}{2\left(1-c_{0}^{2}\right)} \lambda^{2}+\mathcal{O}\left(\lambda^{3}+\lambda^{2} \varepsilon+\lambda \varepsilon^{2}\right),
$$

so that the linearised stability problem (3.13) has two eigenvalues near $0, \lambda=0$ and

$$
\lambda=-\varepsilon\left(\alpha+\frac{1+2 c_{0}^{2}}{1-c_{0}^{2}} \beta\right)+\mathcal{O}\left(\varepsilon^{2}\right)
$$

Proof of Lemma 5.3 As in the previous section, we define $\widetilde{V}^{\varepsilon}(\xi)=\frac{1}{2}\left(u_{\varepsilon},\left(u_{\varepsilon}\right)_{\xi},\left(u_{\varepsilon}\right)_{\xi \xi}\right)^{T}$. Then, $U^{+}(\xi, 0, \varepsilon)=C_{\varepsilon}^{+} \widetilde{V}_{\xi}^{\varepsilon}(\xi)$ and $U^{-}(\xi, 0, \varepsilon)=C_{\varepsilon}^{-} \widetilde{V}_{\xi}^{\varepsilon}(\xi)$, where $C_{\varepsilon}^{ \pm}$are such that the normalisation (3.18) is satisfied. By sections 3.2 and 5.1 we find that $C_{0}^{ \pm}=\frac{1}{2}$. Since $U^{+}$and $U^{-}$are parallel, it follows immediately that $D^{\beta}(0, \varepsilon)=0$.

Next, we calculate the derivative of the Evans function with respect to $\lambda$ at $\lambda=0$

$$
\begin{aligned}
\frac{\partial}{\partial \lambda} D^{\beta}(0, \varepsilon)=e^{-\int_{0}^{\xi}} \operatorname{tr}\left(\mathbf{A}^{\beta}(s, 0, \varepsilon)\right) d s \\
\\
\left(C_{\varepsilon}^{-} \frac{\partial}{\partial \lambda} U^{+}(\xi, 0, \varepsilon) \wedge \widetilde{V}_{\xi}^{\varepsilon}(\xi) \wedge U^{f}(\xi, 0, \varepsilon)\right. \\
\quad+C_{\varepsilon}^{+} \widetilde{V}_{\xi}^{\varepsilon}(\xi) \wedge \frac{\partial}{\partial \lambda} U^{-}(\xi, 0, \varepsilon) \wedge U^{f}(\xi, 0, \varepsilon) \\
\left.\quad+C_{\varepsilon}^{+} C_{\varepsilon}^{-} \widetilde{V}_{\xi}^{\varepsilon}(\xi) \wedge \widetilde{V}_{\xi}^{\varepsilon}(\xi) \wedge \frac{\partial}{\partial \lambda} U^{f}(\xi, 0, \varepsilon)\right) \\
=W^{+}(\xi, \varepsilon)-W^{-}(\xi, \varepsilon)+0,
\end{aligned}
$$

where

$$
W^{ \pm}(\xi, \varepsilon)=C_{\varepsilon}^{\mp} e^{-\int_{0}^{\xi} \operatorname{tr}\left(\mathbf{A}^{\beta}(s, 0, \varepsilon)\right) d s} \frac{\partial}{\partial \lambda} U^{ \pm}(\xi, 0, \varepsilon) \wedge \widetilde{V}_{\xi}^{\varepsilon}(\xi) \wedge U^{f}(\xi, 0, \varepsilon) .
$$

Note that

$$
\operatorname{tr}\left(\mathbf{A}^{\beta}(s, 0, \varepsilon)\right)=\operatorname{tr}\left(\mathbf{A}_{0}^{\beta}(0, \varepsilon)\right)=\mu_{1}(0, \varepsilon)+\mu_{2}(0, \varepsilon)+\mu_{3}(0, \varepsilon) .
$$

Furthermore, $U^{f}$ cannot grow faster than $e^{\mu_{3} \xi}$ for $\xi \rightarrow \operatorname{sgn}\left(\beta c_{\varepsilon}\right) \infty$ and decays like $e^{\mu_{3} \xi}$ for $\xi \rightarrow-\operatorname{sgn}\left(\beta c_{\varepsilon}\right) \infty$. Hence $U^{f} \sim e^{\mu_{3} \xi}$ for $\xi \rightarrow \pm \infty$. Therefore, at $\xi=+\infty$,

$$
\widetilde{V}_{\xi}^{\varepsilon}(\xi) \sim e^{\mu_{1} \xi}, \quad U^{f}(\xi, 0, \varepsilon) \sim e^{\mu_{3} \xi}, \quad \text { and } \quad \frac{\partial}{\partial \lambda} U^{+}(\xi, 0, \varepsilon) \sim e^{\left(\mu_{1}+\delta\right) \xi}
$$

for any $\delta>0$. This implies that

$$
W^{+}(\xi, \varepsilon) \sim e^{-\left(\mu_{1}+\mu_{2}+\mu_{3}\right) \xi} e^{\mu_{1} \xi} e^{\mu_{3} \xi} e^{\left(\mu_{1}+\delta\right) \xi}=e^{\left(-\mu_{2}+\mu_{1}+\delta\right) \xi} \rightarrow 0, \quad \xi \rightarrow+\infty,
$$


since $\mu_{1}<0$ and $\mu_{2}>0$. Similarly, at $\xi=-\infty$,

$$
\widetilde{V}_{\xi}^{\varepsilon}(\xi) \sim e^{\mu_{2} \xi}, \quad U^{f}(\xi, 0, \varepsilon) \sim e^{\mu_{3} \xi}, \quad \text { and } \quad \frac{\partial}{\partial \lambda} U^{-}(\xi, 0, \varepsilon) \sim e^{\left(\mu_{2}-\delta\right) \xi}
$$

for any $\delta>0$. Thus

$$
W^{-}(\xi, \varepsilon) \sim e^{-\left(\mu_{1}+\mu_{2}+\mu_{3}\right) \xi} e^{\mu_{2} \xi} e^{\mu_{3} \xi} e^{\left(\mu_{2}-\delta\right) \xi}=e^{\left(-\mu_{1}+\mu_{2}-\delta\right) \xi} \rightarrow 0, \quad \xi \rightarrow-\infty
$$

since $\mu_{1}<0$ and $\mu_{2}>0$.

We analyse the derivative of $U^{ \pm}$with respect to $\lambda$ in a similar way as in the previous section. By differentiating (3.13) with respect to $\lambda$ and setting $\lambda=0$, we see that $\frac{\partial}{\partial \lambda} U^{ \pm}(\xi, 0, \varepsilon)$ satisfies

$$
\left(\frac{\partial}{\partial \lambda} U^{ \pm}(\xi, 0, \varepsilon)\right)_{\xi}=\mathbf{A}^{\beta}(\xi, 0, \varepsilon) \frac{\partial}{\partial \lambda} U^{ \pm}(\xi, 0, \varepsilon)+C_{\varepsilon}^{ \pm} \mathbf{M}^{\beta}(\varepsilon) \widetilde{V}_{\xi}^{\varepsilon}(\xi)
$$

where

$$
\mathbf{M}^{\beta}(\varepsilon)=\frac{\partial}{\partial \lambda} \mathbf{A}^{\beta}(\xi, 0, \varepsilon)=\frac{1}{\varepsilon}\left(\begin{array}{ccc}
0 & 0 & 0 \\
0 & 0 & 0 \\
-\frac{\varepsilon \alpha\left(\sqrt{1-c_{\varepsilon}^{2}}\right)^{3}}{\beta c_{\varepsilon}} & \frac{2\left(1-c_{\varepsilon}^{2}\right)}{\beta} & \frac{\varepsilon \sqrt{1-c_{\varepsilon}^{2}}}{c_{\varepsilon}}
\end{array}\right) .
$$

Thus, we get for the $\xi$-derivative of $W^{ \pm}(\xi, \varepsilon)$

$$
\begin{aligned}
D_{\xi} W^{ \pm}(\xi, \varepsilon)=- & \operatorname{tr}\left(\mathbf{A}^{\beta}(\xi, 0, \varepsilon)\right) W^{ \pm}(\xi, \varepsilon) \\
& +C_{\varepsilon}^{\mp} e^{-\int_{0}^{\xi} \operatorname{tr}\left(\mathbf{A}^{\beta}(s, 0, \varepsilon)\right) d s} \\
& {\left[\mathbf{A}^{\beta}(\xi, 0, \varepsilon) \frac{\partial}{\partial \lambda} U^{ \pm}(\xi, 0, \varepsilon) \wedge \widetilde{V}_{\xi}^{\varepsilon}(\xi) \wedge U^{f}(\xi, 0, \varepsilon)\right.} \\
& +C_{\varepsilon}^{ \pm} \mathbf{M}^{\beta}(\varepsilon) \widetilde{V}_{\xi}^{\varepsilon}(\xi) \wedge \widetilde{V}_{\xi}^{\varepsilon}(\xi) \wedge U^{f}(\xi, 0, \varepsilon) \\
& +\frac{\partial}{\partial \lambda} U^{ \pm}(\xi, \varepsilon) \wedge \mathbf{A}^{\beta}(\xi, 0, \varepsilon) \widetilde{V}_{\xi}^{\varepsilon}(\xi) \wedge U^{f}(\xi, 0, \varepsilon) \\
& \left.+\frac{\partial}{\partial \lambda} U^{ \pm}(\xi, \varepsilon) \wedge \widetilde{V}_{\xi}^{\varepsilon}(\xi) \wedge \mathbf{A}^{\beta}(\xi, 0, \varepsilon) U^{f}(\xi, 0, \varepsilon)\right] \\
= & C_{\varepsilon}^{\mp} C_{\varepsilon}^{ \pm} e^{-\int_{0}^{\xi} \operatorname{tr}\left(\mathbf{A}^{\beta}(s, 0, \varepsilon)\right) d s} \mathbf{M}^{\beta}(\varepsilon) \widetilde{V}_{\xi}^{\varepsilon}(\xi) \wedge \widetilde{V}_{\xi}^{\varepsilon}(\xi) \wedge U^{f}(\xi, 0, \varepsilon),
\end{aligned}
$$

since of course also $\mathbf{B} U_{1} \wedge U_{2} \wedge U_{3}+U_{1} \wedge \mathbf{B} U_{2} \wedge U_{3}+U_{1} \wedge U_{2} \wedge \mathbf{B} U_{3}=\operatorname{tr}(\mathbf{B}) U_{1} \wedge U_{2} \wedge U_{3}$, for vectors $U_{1,2,3}$ and any matrix $\mathbf{B}$. We conclude by $\lim _{\xi \rightarrow \pm \infty} W^{ \pm}(\xi, \varepsilon)=0$ that

$$
\begin{aligned}
\frac{\partial}{\partial \lambda} D^{\beta}(0, \varepsilon) & =W^{+}(\xi, \varepsilon)-W^{-}(\xi, \varepsilon) \\
& =-C_{\varepsilon}^{\mp} C_{\varepsilon}^{ \pm} \int_{-\infty}^{\infty} e^{-\int_{0}^{\xi} \operatorname{tr}\left(\mathbf{A}^{\beta}(s, 0, \varepsilon)\right) d s} \mathbf{M}^{\beta}(\varepsilon) \widetilde{V}_{\xi}^{\varepsilon}(\xi) \wedge \widetilde{V}_{\xi}^{\varepsilon}(\xi) \wedge U^{f}(\xi, 0, \varepsilon) d \xi
\end{aligned}
$$

where $\operatorname{tr}\left(\mathbf{A}^{\beta}(\xi, 0, \varepsilon)\right)=\frac{\left(\sqrt{1-c_{\varepsilon}^{2}}\right)^{3}}{\varepsilon \beta c_{\varepsilon}}$. Using the definition of the matrix $\mathbf{M}^{\beta}(\varepsilon)(5.4)$ and the explicit expressions for $U^{f}(\xi, 0, \varepsilon)(5.3)$ and $\mu_{3}(0, \varepsilon)(3.16)$, we introduce

$$
\begin{aligned}
E_{\text {tot }}(\xi) \stackrel{\text { def }}{=} & e^{-\operatorname{tr}\left(\mathbf{A}^{\beta}(0, \varepsilon)\right) \xi} \mathbf{M}^{\beta}(\varepsilon) \widetilde{V}_{\xi}^{\varepsilon}(\xi) \wedge \widetilde{V}_{\xi}^{\varepsilon}(\xi) \wedge U^{f}(\xi, 0, \varepsilon) \\
= & e^{\left(\mu_{3}(\varepsilon)-\operatorname{tr}\left(\mathbf{A}^{\beta}(\varepsilon)\right)\right) \xi}\left(\frac{c_{0}}{2 \sqrt{1-c_{0}^{2}}}\left(u_{\varepsilon}\right)_{\xi}\left(u_{\varepsilon}\right)_{\xi \xi}+\varepsilon\left[-\frac{\alpha}{4}\left(\left(u_{\varepsilon}\right)_{\xi}\right)^{2}-\frac{\beta c_{0}^{2}}{2\left(1-c_{0}^{2}\right)^{2}}\left(\left(u_{\varepsilon}\right)_{\xi \xi}\right)^{2}\right.\right. \\
& \left.\left.+\frac{\beta}{4\left(1-c_{0}^{2}\right)}\left(u_{\varepsilon}\right)_{\xi}\left(u_{\varepsilon}\right)_{\xi \xi \xi}+\frac{\beta c_{0}^{2}}{\left(1-c^{2}\right)^{2}}\left(\tanh \xi+\frac{\beta c_{0}}{\beta c_{0} \mid}\right)\left(u_{\varepsilon}\right)_{\xi \xi}\left(u_{\varepsilon}\right)_{\xi}\right]\right)+\mathcal{O}\left(\varepsilon^{2}\right) \\
\stackrel{\text { def }}{=} & E_{\mathcal{O}(1)}(\xi)+\varepsilon\left[E_{\alpha}(\xi)+E_{\beta, 1}(\xi)+E_{\beta, 2}(\xi)+E_{\tanh }(\xi)\right]+\mathcal{O}\left(\varepsilon^{2}\right) .
\end{aligned}
$$


Since $\left(u_{0}\right)_{\xi}(\xi)=2 \operatorname{sech} \xi$, we find

$$
\begin{aligned}
\int_{-\infty}^{\infty} E_{\alpha}(\xi) d \xi & =-\alpha \int_{-\infty}^{\infty} \operatorname{sech}^{2} \xi d \xi+\mathcal{O}(\varepsilon)=-2 \alpha+\mathcal{O}(\varepsilon) \\
\int_{-\infty}^{\infty} E_{\beta, 1}(\xi) d \xi & =-\frac{2 \beta c_{0}^{2}}{\left(1-c_{0}^{2}\right)^{2}} \int_{-\infty}^{\infty} \tanh ^{2} \xi \operatorname{sech}^{2} \xi d \xi+\mathcal{O}(\varepsilon)=-\frac{4 \beta c_{0}^{2}}{3\left(1-c_{0}^{2}\right)^{2}}+\mathcal{O}(\varepsilon), \\
\int_{-\infty}^{\infty} E_{\beta, 2}(\xi) d \xi & =-\frac{\beta}{4\left(1-c_{0}^{2}\right)} \int_{-\infty}^{\infty}\left(\left(u_{\varepsilon}\right)_{\xi \xi}\right)^{2} d \xi+\mathcal{O}(\varepsilon)=-\frac{2 \beta}{3\left(1-c_{0}^{2}\right)}+\mathcal{O}(\varepsilon) \\
\int_{-\infty}^{\infty} E_{\tanh }(\xi) d \xi & =-\frac{4 \beta c_{0}^{2}}{\left(1-c_{0}^{2}\right)^{2}} \int_{-\infty}^{\infty} \tanh ^{2} \xi \operatorname{sech}^{2} \xi d \xi+\mathcal{O}(\varepsilon)=-\frac{8 \beta c_{0}^{2}}{3\left(1-c_{0}^{2}\right)^{2}}+\mathcal{O}(\varepsilon), \\
\text { and } & \\
\int_{-\infty}^{\infty} E_{\mathcal{O}(1)}(\xi) d \xi & =-\frac{c_{0}}{4 \sqrt{1-c_{0}^{2}}} \int_{-\infty}^{\infty}\left(\left(u_{\varepsilon}\right)_{\xi}\right)^{2} \frac{d}{d \xi}\left(e^{\left(\mu_{3}-\operatorname{tr}\left(\mathbf{A}^{\beta}\right)\right) \xi}\right) d \xi=-\frac{2 \varepsilon\left(\alpha\left(1-c_{0}^{2}\right)-\beta\right) c_{0}^{2}}{\left(1-c_{0}^{2}\right)^{2}}+\mathcal{O}\left(\varepsilon^{2}\right)
\end{aligned}
$$

Hence, by $C_{\varepsilon}^{ \pm}=\frac{1}{2}+\mathcal{O}(\varepsilon)$,

$$
\frac{\partial}{\partial \lambda} D^{\beta}(0, \varepsilon)=\int_{-\infty}^{\infty} E_{\text {tot }}(\xi) d \xi=\frac{3 \alpha\left(1-c_{0}^{2}\right)+\left(1+2 c_{0}^{2}\right) \beta}{6\left(1-c_{0}^{2}\right)^{2}} \varepsilon+\mathcal{O}\left(\varepsilon^{2}\right) .
$$

Remark 5.5 As for the existence problem, we can conclude that the singular perturbation term $\varepsilon \beta u_{x x t}$ in (1.1) has a regular effect on $\frac{\partial}{\partial \lambda} D^{\beta}(0, \varepsilon)$. On the other hand, however, only the contributions to $\frac{\partial}{\partial \lambda} D^{\beta}(0, \varepsilon)$ through the components $E_{\alpha}(\xi)$ and $E_{\beta, 2}(\xi)$ appear from "regular" interactions of leading order terms (with respect to $\varepsilon$ ). The other three leading order contributions, through $E_{\mathcal{O}(1)}(\xi), E_{\beta, 1}(\xi)$ and $E_{\text {tanh }}(\xi)$, have their origin in higher order effects that one would a priori not incorporate in the perturbation analysis (the leading order contribution to the integral over $E_{\mathcal{O}(1)}(\xi)$ comes from the third term in the expansion of $\mu_{3}(0, \varepsilon)(3.16) ; E_{\beta, 1}(\xi)$ and $E_{\tanh }(\xi)$ originate from $\mathcal{O}\left(\varepsilon^{2}\right)$ terms in $\left.U^{f}(\xi, 0, \varepsilon)(5.3)\right)$.

\subsection{The Evans function for $\lambda$ near $\pm \mathrm{i} \omega_{0}$ and $\varepsilon$ near zero}

Instability can also occur via eigenvalues bifurcating from the endpoints of the continuous spectrum, i.e., from $\lambda= \pm \mathrm{i} \omega_{0}$. As in the case with no surface resistance, an expansion of the Evans function will be derived for $\lambda$ near $\pm \mathrm{i} \omega_{0}$ and $\varepsilon$ small. Again, we can restrict the analysis to the case $\lambda=\mathrm{i} \omega_{0}$.

Recall the characteristic polynomial (3.15). For $\varepsilon$ small, a double eigenvalue occurs if the two slow eigenvalues collide, i.e., at $\lambda=\mathrm{i} \omega_{0}+\widetilde{\lambda}(\varepsilon)$, where $\widetilde{\lambda}(\varepsilon)=\widetilde{\lambda}^{+}(\varepsilon)$ is given in (3.19). If we let $\sigma=\lambda-\widetilde{\lambda}(\varepsilon)$ and define the matrix $\widehat{\mathbf{A}}^{\beta}(\xi, \sigma, \varepsilon)=\mathbf{A}^{\beta}(\xi, \sigma+\widetilde{\lambda}(\varepsilon), \varepsilon)$ and the Evans function $\widehat{D}(\sigma, \varepsilon)=D^{\beta}(\sigma+\widetilde{\lambda}(\varepsilon), \varepsilon)$, then the matrix

$$
\widehat{\mathbf{A}}_{0}^{\beta}(\sigma, \varepsilon)=\widehat{\mathbf{A}}^{\beta}( \pm \infty, \sigma, \varepsilon)=\mathbf{A}_{0}^{\beta}(\sigma+\widetilde{\lambda}(\varepsilon), \varepsilon)
$$

has an eigenvalue

$$
\mu_{d}(\varepsilon)=-\mathrm{i} c_{0}-\frac{\varepsilon \beta c_{0}}{\left(\sqrt{1-c_{0}^{2}}\right)^{3}}+\mathcal{O}\left(\varepsilon^{2}\right)
$$

at $\sigma=\mathrm{i} \omega_{0}$ of algebraic multiplicity 2 and geometric multiplicity 1 . In a similar way as before, we let $U^{ \pm}(\xi, \varepsilon)$ be slow solutions of the linearised system (3.13) at $\sigma=\mathrm{i} \omega_{0}$ that are 
determined by their behaviour at $\xi= \pm \infty$ (3.18). In section 3 , we have seen that for $\varepsilon=0$ the linearised solutions are

$$
U^{+}(\xi, 0)=-U^{-}(\xi, 0)=e^{-i c_{0} \xi}\left(\begin{array}{c}
\tanh \xi \\
-\mathrm{i} c_{0} \tanh \xi+\operatorname{sech}^{2} \xi \\
-c_{0}^{2} \tanh \xi-2 \mathrm{i} c_{0} \operatorname{sech}^{2} \xi-2 \operatorname{sech}^{2} \xi \tanh \xi
\end{array}\right)
$$

Note that $U^{ \pm}(\xi, 0)$ satisfy the normalisation (3.18).

In the next Lemma we will prove that up to first order, the Evans function $\widehat{D}\left(\mathrm{i} \omega_{0}, \varepsilon\right)$ will remain on the imaginary axis.

Lemma 5.6 The Evans function satisfies $\hat{D}\left(\sigma_{0}, 0\right)=0$ and $\frac{\partial}{\partial \varepsilon} \hat{D}\left(\sigma_{0}, 0\right)=-\frac{4}{3} i \beta \frac{1+2 c_{0}^{2}}{\left(\sqrt{1-c_{0}^{2}}\right)^{3}}$.

Before proving this Lemma, we first look at its implication for the bifurcation of eigenvalues out of the continuous spectrum. There is a $\delta>0$ such that for $U_{\delta}$

$$
U_{\delta}=\left\{\sigma \in \mathbb{C}|| \sigma-\mathrm{i} \omega_{0} \mid<\delta\right\} \backslash\left\{\sigma \in \mathbb{C} \mid \Re(\sigma)<0, \Im(\sigma)=\omega_{0}\right\}
$$

the Evans function $\widehat{D}(\sigma, \varepsilon)$ is continuous for $\sigma \in U_{\delta}$ and $\varepsilon$ near 0 and $\widehat{D}\left(\mathrm{i} \omega_{0}, \varepsilon\right)$ is continuously differentiable in $\varepsilon$ for $\varepsilon$ near 0 (see section 4 of [11]). Hence for $\sigma \in U_{\varepsilon}$, and $\varepsilon$ small, there exists some function $R(\sigma, \varepsilon)$ such that

$$
\widehat{D}(\sigma, \varepsilon)=\widehat{D}(\sigma, 0)+\varepsilon \frac{\partial}{\partial \varepsilon} \widehat{D}\left(\mathrm{i} \omega_{0}, 0\right)+\varepsilon R(\sigma, \varepsilon),
$$

and $R$ is continuous with $R\left(\mathrm{i} \omega_{0}, 0\right)=0$, hence $R(\sigma, \varepsilon)=o(1)$. Using the expressions for the unperturbed Evans function and the derivative of the Evans function, we get that

$$
\widehat{D}(\sigma, \varepsilon)=\frac{2 \sigma^{2}}{1-c_{0}^{2}} \frac{\sqrt{\sigma+\mathrm{i} \omega_{0}} \sqrt{\sigma-\mathrm{i} \omega_{0}}}{\omega_{0}}-\frac{4}{3} i \beta \frac{1+2 c_{0}^{2}}{\left(\sqrt{1-c_{0}^{2}}\right)^{3}} \varepsilon+\varepsilon R(\sigma, \varepsilon) .
$$

For $\sigma$ near $\mathrm{i} \omega_{0}$, we have that

$$
\widehat{D}(\sigma, 0)=-2(1+\mathrm{i})\left(1-c_{0}^{2}\right)^{-\frac{1}{4}} \sqrt{\sigma-\mathrm{i} \omega_{0}}+\mathcal{O}\left(\sigma-\mathrm{i} \omega_{0}\right)
$$

(Lemma 3.4). Hence with the given branch cut for the definition of the square root (3.12), we get that $\frac{3 \pi}{4}<\arg \widehat{D}(\sigma, 0) \leq \frac{7 \pi}{4}$. Since for $\beta>0, \arg \left(\frac{4}{3} i \beta \frac{1+2 c_{0}^{2}}{\left(\sqrt{1-c_{0}^{2}}\right)^{3}}\right)=\frac{\pi}{2}$, we conclude that $\widehat{D}(\sigma, \varepsilon) \neq 0$ for $\varepsilon$ small and $\sigma$ near $\mathrm{i} \omega_{0}$. Hence for $\beta>0$, no eigenvalue will bifurcate out of the continuous spectrum.

Remark 5.7 The Evans function $D^{\beta}(\lambda, \varepsilon)$ can also be interpreted as being defined on a Riemann surface $\mathcal{R}[8,11,6]$. Eigenvalues of the associated linearised stability problem (3.14) correspond to zeroes of $D^{\beta}(\lambda, \varepsilon)$ on one of the sheets of $\mathcal{R}$ (that is determined by the branch cut). The Evans function can also have zeroes on another sheet of $\mathcal{R}$, but these zeroes have no direct relevance for the stability problem. Lemma 5.6 establishes the existence of a (complex conjugate) pair of 'irrelevant' zeroes of $D^{\beta}(\lambda, \varepsilon)$ on $\mathcal{R}$.

Proof of Lemma 5.6 Since $U^{+}(\xi, 0)=-U^{-}(\xi, 0), \mu_{d}(0)=-\mathrm{i} c_{0}$ and 


$$
\lim _{\varepsilon \rightarrow 0} e^{-\mu_{3}(\lambda, \varepsilon) \xi} U^{f}(\xi, \varepsilon)=(0,0,1) \text { at } \sigma=i \omega_{0}, \text { we see immediately that } \widehat{D}\left(\mathrm{i} \omega_{0}, 0\right)=0 \text {. }
$$

To analyse the derivative of the Evans function we define

$$
\widehat{U}^{f}(\xi, \varepsilon)=e^{-\widehat{\mu}_{3}\left(\mathrm{i} \omega_{0}, \varepsilon\right) \xi} U^{f}\left(\xi, \mathrm{i} \omega_{0}+\widetilde{\lambda}(\varepsilon), \varepsilon\right),
$$

where $\left.\widehat{\mu}_{3}(\sigma, \varepsilon)=\mu_{3}(\sigma+\widetilde{\lambda}(\varepsilon), \varepsilon)\right)$. With (5.3), it follows that

$$
\widehat{U}^{f}(\xi, \varepsilon)=\left(\begin{array}{c}
\mathcal{O}\left(\varepsilon^{2}\right) \\
\varepsilon \frac{\beta c_{0}}{\left(\sqrt{1-c_{0}^{2}}\right)^{3}}+\mathcal{O}\left(\varepsilon^{2}\right) \\
1+\mathcal{O}(\varepsilon)
\end{array}\right)
$$

Furthermore,

$$
\frac{\partial}{\partial \xi} \widehat{U}^{f}(\xi, \varepsilon)=\left(\widehat{A}^{\beta}\left(\xi, \mathrm{i} \omega_{0}, \varepsilon\right)-\widehat{\mu}_{3}\left(\mathrm{i} \omega_{0}, \varepsilon\right)\right) \widehat{U}^{f}(\xi, \varepsilon) .
$$

With the definition of $\widehat{U}^{f}(\xi, \varepsilon)$, the Evans function at $\sigma=\mathrm{i} \omega_{0}$ can be written as

$$
\widehat{D}\left(\mathrm{i} \omega_{0}, \varepsilon\right)=e^{-2 \mu_{d}(\varepsilon) \xi} U^{+}(\xi, \varepsilon) \wedge U^{-}(\xi, \varepsilon) \wedge \widehat{U}^{f}(\xi, \varepsilon) .
$$

Using that $U^{+}(\xi, 0)=-U^{-}(\xi, 0)$ and that $\lim _{\varepsilon \rightarrow 0} \frac{\partial}{\partial \varepsilon} \widehat{U}^{f}(\xi, \varepsilon)$ is well-defined, we get

$$
\begin{aligned}
\frac{\partial}{\partial \varepsilon} \widehat{D}\left(\mathrm{i} \omega_{0}, 0\right) & =e^{-2 \mu_{d}(0) \xi}\left(\frac{\partial}{\partial \varepsilon} U^{+} \wedge U^{-} \wedge \widehat{U}^{f}+U^{+} \wedge \frac{\partial}{\partial \varepsilon} U^{-} \wedge \widehat{U}^{f}\right)(\xi, 0) \\
& =\left(W^{+}-W^{-}\right)(\xi, 0)
\end{aligned}
$$

where

$$
W^{ \pm}(\xi, \varepsilon)=e^{-2 \mu_{d}(\varepsilon) \xi}\left(\frac{\partial}{\partial \varepsilon} U^{ \pm} \wedge U^{\mp} \wedge \widehat{U}^{f}\right)(\xi, \varepsilon) .
$$

As both $U^{ \pm}(\xi, \varepsilon)$ are solutions of the linear equation $U_{\xi}^{ \pm}=\widehat{\mathbf{A}}^{\beta} U^{ \pm}$, we find by differentiation with respect to $\varepsilon$ that

$$
\frac{\partial^{2} U^{ \pm}}{\partial \xi \partial \varepsilon}(\xi, \varepsilon)=\frac{\partial\left(\widehat{\mathbf{A}}^{\beta}\right)}{\partial \varepsilon}\left(\xi, \mathrm{i} \omega_{0}, \varepsilon\right) U^{ \pm}(\xi, \varepsilon)+\widehat{\mathbf{A}}^{\beta}\left(\xi, \mathrm{i} \omega_{0}, \varepsilon\right) \frac{\partial U^{ \pm}}{\partial \varepsilon}(\xi, \varepsilon) .
$$

Note that $\frac{\partial\left(\widehat{\mathbf{A}}^{\beta}\right)}{\partial \varepsilon}\left(\xi, \mathrm{i} \omega_{0}, \varepsilon\right)$ and $\left(\widehat{\mathbf{A}}^{\beta}\right)\left(\xi, \mathrm{i} \omega_{0}, \varepsilon\right)$ cannot be evaluated directly at $\varepsilon=0$. Using (5.6), we get

$$
\begin{aligned}
\frac{\partial}{\partial \xi} W^{ \pm}(\xi, \varepsilon)= & -2 \mu_{d}(\varepsilon) W^{ \pm}(\xi, \varepsilon)+e^{-2 \mu_{d}(\varepsilon) \xi}\left(\left[\frac{\partial \widehat{\mathbf{A}}^{\beta}}{\partial \varepsilon} U^{ \pm}+\widehat{\mathbf{A}}^{\beta} \frac{\partial U^{ \pm}}{\partial \varepsilon}\right] \wedge U^{\mp} \wedge \widehat{U}^{f}\right. \\
& \left.+\frac{\partial U^{ \pm}}{\partial \varepsilon} \wedge \widehat{\mathbf{A}}^{\beta} U^{\mp} \wedge \widehat{U}^{f}+\frac{\partial U^{ \pm}}{\partial \varepsilon} \wedge U^{\mp} \wedge\left[\widehat{\mathbf{A}}^{\beta}-\widehat{\mu}_{3}\right] \widehat{U}^{f}\right)(\xi, \varepsilon) .
\end{aligned}
$$

Hence we conclude that

$$
\begin{aligned}
\frac{\partial}{\partial \xi} W^{ \pm}(\xi, \varepsilon)= & {\left[\operatorname{trace}\left(\widehat{\mathbf{A}}^{\beta}\left(\xi, \mathrm{i} \omega_{0}, \varepsilon\right)\right)-\left(2 \mu_{d}(\varepsilon)+\widehat{\mu}_{3}\left(\mathrm{i} \omega_{0}, \varepsilon\right)\right)\right] W^{ \pm}(\xi, \varepsilon)+} \\
& e^{-2 \mu_{d}(\varepsilon) \xi}\left(\frac{\partial \widehat{\mathbf{A}}^{\beta}}{\partial \varepsilon}\left(\xi, \mathrm{i} \omega_{0}, \varepsilon\right) U^{ \pm} \wedge U^{\mp} \wedge \widehat{U}^{f}\right)(\xi, \varepsilon) \\
= & e^{-2 \mu_{d}(\varepsilon) \xi}\left(\frac{\partial \widehat{\mathbf{A}}^{\beta}}{\partial \varepsilon}\left(\xi, \mathrm{i} \omega_{0}, \varepsilon\right) U^{ \pm} \wedge U^{\mp} \wedge \widehat{U}^{f}\right)(\xi, \varepsilon)
\end{aligned}
$$


Altogether we have arrived at the following expression:

$$
\begin{aligned}
\frac{\partial}{\partial \varepsilon} \widehat{D}\left(\mathrm{i} \omega_{0}, 0\right)=\lim _{\varepsilon \rightarrow 0} & -\int_{0}^{\infty} e^{-2 \mu_{d}(\varepsilon) \xi}\left(\frac{\partial \widehat{\mathbf{A}}}{\partial \varepsilon} U^{+} \wedge U^{-} \wedge \widehat{U}^{f}\right)(\xi, \varepsilon) d \xi \\
& \left.-\int_{-\infty}^{0} e^{-2 \mu_{d}(\varepsilon) \xi}\left(\frac{\partial \widehat{\mathbf{A}}}{\partial \varepsilon} U^{-} \wedge U^{+} \wedge \widehat{U}^{f}\right)(\xi, \varepsilon) d \xi\right) \\
+ & W^{+}(\infty, 0)-W^{-}(-\infty, 0),
\end{aligned}
$$

where

$$
W^{ \pm}( \pm \infty, 0)=\lim _{\xi \rightarrow \pm \infty} e^{-2 \mu_{d}(0) \xi}\left(\frac{\partial U^{ \pm}}{\partial \varepsilon} \wedge U^{\mp} \wedge \widehat{U}^{f}\right)(\xi, 0) .
$$

Unlike in the previous cases, these terms do not vanish. However, it can be shown that $W^{+}(\infty, 0)-W^{-}(-\infty, 0)=0$. It follows from the normalisation (3.18) that

$$
\lim _{\xi \rightarrow \pm \infty} e^{-\mu_{d}(\varepsilon) \xi} U^{ \pm}(\xi, \varepsilon)=\eta_{d}(\varepsilon)=\left(1, \mu_{d}(\varepsilon), \mu_{d}(\varepsilon)^{2}\right)^{T} .
$$

In other words, there exists some function $V^{ \pm}(\xi, \varepsilon)$ such that $U^{ \pm}(\xi, \varepsilon)=e^{\mu_{d}(\varepsilon) \xi}\left(\eta_{d}(\varepsilon)+V^{ \pm}(\xi, \varepsilon)\right)$ and that $\lim _{\xi \rightarrow \pm \infty} V^{ \pm}(\xi, \varepsilon)=0$. This implies that $\lim _{\xi \rightarrow \pm \infty} \frac{\partial}{\partial \varepsilon} V^{ \pm}(\xi, \varepsilon)=0$ and

$$
\frac{\partial U^{ \pm}}{\partial \varepsilon}(\xi, 0)=e^{\mu_{d}(0) \xi}\left(\xi \frac{\partial \mu_{d}}{\partial \varepsilon}(0)\left(\eta_{d}(0)+V^{ \pm}(\xi, 0)\right)+\left(\frac{\partial \eta_{d}}{\partial \varepsilon}(0)+\frac{\partial V^{ \pm}}{\partial \varepsilon}(\xi, 0)\right)\right)
$$

Using that $U^{+}(\xi, 0)=-U^{-}(\xi, 0)$, hence $V^{ \pm}(\xi, 0)=-2 \eta_{d}(0)-V^{\mp}(\xi, 0)$, this implies that

$$
e^{-2 \mu_{d}(0) \xi}\left(\frac{\partial U^{ \pm}}{\partial \varepsilon} \wedge U^{\mp}\right)(\xi, 0)=\left(\frac{\partial \eta_{d}}{\partial \varepsilon}(0)+\frac{\partial V^{ \pm}}{\partial \varepsilon}(\xi, 0)\right) \wedge\left(-\eta_{d}(0)-V^{ \pm}(\xi, 0)\right)+0 .
$$

Since $\lim _{\xi \rightarrow \pm \infty} \frac{\partial V^{ \pm}}{\partial \varepsilon}(\xi, \varepsilon)=0, \lim _{\xi \rightarrow \pm \infty} V^{ \pm}(\xi, \varepsilon)=0$, and $\lim _{\xi \rightarrow \pm \infty} \widehat{U}^{f}(\xi, 0)=(0,0,1)^{T}=\widehat{U}^{f}(0,0)$ (5.3), we find

$$
W^{ \pm}( \pm \infty, 0)=-\frac{\partial \eta_{d}}{\partial \varepsilon}(0) \wedge \eta_{d}(0) \wedge \widehat{U}^{f}(0,0)=\mu_{d}^{\prime}(0)=-\frac{\beta c_{0}}{\left(\sqrt{1-c_{0}^{2}}\right)^{3}},
$$

so that $W^{+}(\infty, 0)=W^{-}(-\infty, 0)$. Thus, by $(5.7)$, our main task is now to determine

$$
F^{ \pm}\left(\xi, \mathrm{i} \omega_{0}, 0\right) \stackrel{\text { def }}{=} \lim _{\varepsilon \rightarrow 0}\left(\frac{\partial \widehat{\mathbf{A}}^{\beta}}{\partial \varepsilon}\left(\xi, \mathrm{i} \omega_{0}, \varepsilon\right) U^{ \pm}(\xi, \varepsilon) \wedge U^{\mp}(\xi, \varepsilon) \wedge \widehat{U}^{f}(\xi, \varepsilon)\right) .
$$

We introduce $\hat{a}_{j}(\xi, \sigma, \varepsilon)(j=1,2,3)$ by

$$
\widehat{\mathbf{A}}^{\beta}(\xi, \sigma, \varepsilon)=\left(\begin{array}{ccc}
0 & 1 & 0 \\
0 & 0 & 1 \\
\frac{\hat{a}_{1}\left(\sqrt{1-c_{0}^{2}}\right)^{3}}{\varepsilon \beta c_{0}} & \frac{\hat{a}_{2}\left(\sqrt{1-c_{0}^{2}}\right)^{3}}{\varepsilon \beta c_{0}} & \frac{\hat{a}_{3}\left(\sqrt{1-c_{0}^{2}}\right)^{3}}{\varepsilon \beta c_{0}}
\end{array}\right),
$$

so that by (3.14) and (3.19)

$$
\begin{aligned}
& \hat{a}_{1}(\xi, \sigma, \varepsilon)=\hat{a}_{10}+\varepsilon \hat{a}_{11}+\mathcal{O}\left(\varepsilon^{2}\right)=-\left(\sigma^{2}+\cos u_{0}(\xi)\right)+\varepsilon\left(\frac{\sigma c_{0}^{2} \beta}{1-c_{0}^{2}}+u_{1}(\xi) \sin u_{0}(\xi)\right)+\mathcal{O}\left(\varepsilon^{2}\right), \\
& \hat{a}_{2}(\xi, \sigma, \varepsilon)=\hat{a}_{20}+\varepsilon \hat{a}_{21}+\mathcal{O}\left(\varepsilon^{2}\right)=2 \frac{\sigma c_{0}}{\sqrt{1-c_{0}^{2}}}-\varepsilon \frac{\beta c_{0}^{3}}{\left(\sqrt{1-c_{0}^{2}}\right)^{3}}+\mathcal{O}\left(\varepsilon^{2}\right), \\
& \hat{a}_{3}(\xi, \sigma, \varepsilon)=\hat{a}_{30}+\varepsilon \hat{a}_{31}+\mathcal{O}\left(\varepsilon^{2}\right)=1+\frac{\sigma \beta}{1-c_{0}^{2}} \varepsilon+\mathcal{O}\left(\varepsilon^{2}\right) .
\end{aligned}
$$


Note that we have again used extensively that $c_{\varepsilon}=c_{0}+\mathcal{O}\left(\varepsilon^{2}\right)$. Due to the structure of $\widehat{\mathbf{A}}^{\beta}(\xi, \sigma, \varepsilon)$, we can write as $U^{ \pm}(\xi, \sigma, \varepsilon)=\left(v^{ \pm}, v_{\xi}^{ \pm}, v_{\xi \xi}^{ \pm}\right)(\xi, \sigma, \varepsilon)$ where $v^{ \pm}(\xi, \sigma, \varepsilon)$ is a solution of the singularly perturbed equation

$$
\hat{a}_{1}(\xi, \varepsilon) v+\hat{a}_{2}(\xi, \varepsilon) v_{\xi}+\hat{a}_{3}(\xi, \varepsilon) v_{\xi \xi}=\varepsilon \frac{\beta c_{0}}{\left(\sqrt{1-c_{0}^{2}}\right)^{3}} v_{\xi \xi \xi}
$$

We are interested in the higher order corrections to the (regular) solutions $U^{ \pm}(\xi, 0)$ at $\sigma=\mathrm{i} \omega_{0}$ given in (5.5). Thus, we expand $v(\xi)=v_{0}(\xi)+\varepsilon v_{1}(\xi)+\mathcal{O}\left(\varepsilon^{2}\right)$ and substitute this into (5.9):

$$
\begin{aligned}
& \hat{a}_{10} v_{0}+\hat{a}_{20} v_{0, \xi}+\hat{a}_{30} v_{0, \xi \xi}=0 \\
& \hat{a}_{10} v_{1}+\hat{a}_{20} v_{1, \xi}+\hat{a}_{30} v_{1, \xi \xi}=H(\xi)=-\hat{a}_{11} v_{0}-\hat{a}_{21} v_{0, \xi}-\hat{a}_{31} v_{0, \xi \xi}+\frac{\beta c_{0}}{\left(\sqrt{\left.1-c_{0}^{2}\right)^{3}} v_{0, \xi \xi \xi} .\right.}
\end{aligned}
$$

Note that $v_{0}^{ \pm}= \pm \tanh \xi e^{-\mathrm{i} c_{0} \xi}$ at $\sigma=\mathrm{i} \omega_{0}(5.5)$, so that also $v_{1}^{+}(\xi)=-v_{1}^{-}(\xi)$. We can now compute $\frac{\partial \widehat{\mathbf{A}}^{\beta}}{\partial \varepsilon}\left(\xi, \mathrm{i} \omega_{0}, \varepsilon\right) U^{ \pm}(\xi, \varepsilon)$,

$$
-\frac{\left(\sqrt{1-c_{0}^{2}}\right)^{3}}{\varepsilon^{2} \beta c_{0}}\left(\begin{array}{ccc}
0 & 0 & 0 \\
0 & 0 & 0 \\
\hat{a}_{10}+\mathcal{O}\left(\varepsilon^{2}\right) & \hat{a}_{20}+\mathcal{O}\left(\varepsilon^{2}\right) & \hat{a}_{30}+\mathcal{O}\left(\varepsilon^{2}\right)
\end{array}\right) U^{ \pm}=\left(\begin{array}{c}
0 \\
0 \\
-\frac{\left(\sqrt{1-c_{0}^{2}}\right)^{3}}{\varepsilon \beta c_{0}} H+\mathcal{O}(1)
\end{array}\right)
$$

which implies (5.8)

$$
\begin{aligned}
F^{ \pm}\left(\xi, \mathrm{i} \omega_{0}, 0\right) & =\left(\hat{a}_{11} v_{0}^{ \pm}+\hat{a}_{21} v_{0, \xi}^{ \pm}+\hat{a}_{31} v_{0, \xi \xi}^{ \pm}-\frac{\beta c_{0}}{\left(\sqrt{1-c_{0}^{2}}\right)^{3}} v_{0, \xi \xi \xi}^{ \pm}\right) v_{0}^{\mp} \\
& =\left(-\left(\frac{\mathrm{i} c_{0}^{2} \beta}{\sqrt{1-c_{0}^{2}}}+u_{1} \sin u_{0}\right) v_{0}^{+}+\frac{\beta c_{0}^{3}}{\left(\sqrt{1-c_{0}^{2}}\right)^{3}} v_{0, \xi}^{+}-\frac{\mathrm{i} \beta}{\sqrt{1-c_{0}^{2}}} v_{0, \xi \xi}^{+}+\frac{\beta c_{0}}{\left(\sqrt{1-c_{0}^{2}}\right)^{3}} v_{0, \xi \xi \xi}^{+}\right) v_{0}^{+} .
\end{aligned}
$$

Thus, by (5.7) and the fact that $\tanh \xi$ is odd and $u_{1}(\xi)\left(=\left.\frac{\partial u_{\varepsilon}(\xi)}{\partial \varepsilon}\right|_{\varepsilon=0}\right)$ even as function of $\xi$ (Lemma 2.3)

$$
\begin{aligned}
\frac{\partial}{\partial \varepsilon} \widehat{D}\left(\mathrm{i} \omega_{0}, 0\right) & =-\int_{-\infty}^{\infty} F^{ \pm}\left(\xi, \mathrm{i} \omega_{0}, 0\right) e^{2 \mathrm{i} c_{0} \xi} d \xi=-2 \mathrm{i} \beta \frac{1+2 c_{0}^{2}}{\left(\sqrt{1-c_{0}^{2}}\right)^{3}} \int_{-\infty}^{\infty} \tanh ^{2} \xi \operatorname{sech}^{2} \xi d \xi \\
& =-\frac{4}{3} \mathrm{i} \beta \frac{1+2 c_{0}^{2}}{\left(\sqrt{1-c_{0}^{2}}\right)^{3}} .
\end{aligned}
$$

\section{Acknowledgement}

This work is partly supported by British Council/NWO travel grants (JRP587, JRP668) and an NWO visitors grant B 61-506. We would like to thank Christopher Jones for the reference to nonlinear stability results in [2].

\section{References}

[1] J.C. Alexander, R. Gardner, and C.K.R.T. Jones. A topological invariant arising in the stability analysis of travelling waves. J. Reine Angew. Math., 410:167-212, 1990. 
[2] P.W. Bates and C.K.R.T. Jones. Invariant manifolds for semilinear partial differential equations. Dynamics Reported, 2:1, 1989.

[3] A. Barone and G. Paternò. Physics and Applications of the Josephson effect. WileyInterscience, 1982.

[4] T.J. Bridges and G. Derks. Constructing the symplectic Evans matrix using maximallyanalytic individual vectors. To appear: Proc. Roy. Soc. Edinburgh, 2002.

[5] A. Doelman, R. A. Gardner, and T.J Kaper. Large stable pulse solutions in reactiondiffusion equations. Indiana Univ. Math. J. 50(1):443-507, 2001.

[6] A. Doelman, R. A. Gardner, and T.J Kaper. A stability index analysis of 1-D patterns of the Gray-Scott model. Memoirs of the AMS 155:737, 2002.

[7] N. Fenichel. Geometric singular perturbation theory for ordinary differential equations. J. Diff. Eq., 31:53-98, 1979.

[8] R. A. Gardner and K. Zumbrun. The gap lemma and geometric criteria for instability of viscous shock profiles. Comm. Pure Appl. Math. 51:797-855, 1998.

[9] N. Grønbech-Jensen, M.R. Samuelsen, P.S. Lomdahl and J.A. Blackburn. Bunched soliton states in weakly coupled sine-Gordon systems. Physical Review B, 42(7):3976-3980, 1990.

[10] J. Guckenheimer and P. Holmes. Nonlinear oscillations, dynamical systems and bifurcations of vector fields. Springer-Verlag, New York, 1983.

[11] T. Kapitula and B. Sandstede. Stability of bright solitary-wave solutions to perturbed nonlinear Schrödinger equations. Physica D 124:58-103, 1998.

[12] T. Kapitula The Evans function and generalized Melnikov integrals. SIAM J. Math. Anal. 30(2):273-297, 1999.

[13] T. Kapitula and B. Sandstede. Edge bifurcations for near integrable systems via Evans function techniques. SIAM J. Math. Anal. 5:1117-1143, 2002.

[14] V.P. Koshelets, S.V. Shitov, L.V. Filippenko, A.M. Baryshev, H. Golstein, T. de Graauw, W. Luinge, H. Schaeffer, and H. van de Stadt. First implementation of a superconducting integrated receiver at $450 \mathrm{GHz}$. Applied Physics Letters, 68(9):1273-1275, 1996.

[15] K. Lonngren and A. Scott, editors. Solitons in Action. Academic Press, Inc., 1978.

[16] K. Maginu. Stability of travelling wave solutions of the active Josephson junction transmission line. Journal of Differential Equations 37:238-260, 1980.

[17] E. Mann. Systematic perturbation theory for sine-Gordon solitons without use of inverse scattering methods. J. Phys. A: Math. Gen. 30:1227-1241, 1997.

[18] D.W. McLaughlin and A.C. Scott. Perturbation analysis of fluxon dynamics. Physical Review A 18(4):1652-1679, 1978. 
[19] R.D. Parmentier. Fluxons in long Josephson junctions. In Lonngren and Scott [15], pages 173-199.

[20] N.F. Pedersen. Solitons in Josephson transmission lines. In Trullinger et al. [24].

[21] G.P. Pepe, R. Scaldaferri, et al. Low- $t_{c}$ three-terminal structures for new superconducting devices. Superconductor Science and Technology, 14:987-993, 2001.

[22] R.L. Pego and M.L. Weinstein. Eigenvalues, and instabilities of solitary waves. Phil. Trans. Roy. Soc. Lond. A, 340:47-94, 1992.

[23] A.C. Scott, F.Y.F Chu and S.A. Reible. Magnetic flux propagation on a Josephson transmission line. J. Appl. Physics, 47:3272-3286, 1976.

[24] S.E. Trullinger, V.E. Zakharov, V.L. Pokrovsky, editors. Solitons. Elsevier Science Publishers, B.V., 1986.

[25] A.V. Ustinov, T. Doderer, R.P. Huebener, N.F. Pedersen, B. Mayer, and V.A. Oboznov. Dynamics of Sine-Gordon solitons in the annular Josephson junction. Phys. Rev. Lett. 69(12):1815-1818, 1992 .

[26] T.P.P. Visser. Modelling and Analysis of long Josephson junctions. PhD Thesis, University of Twente, 2002.

[27] Y. Zhang and D. Gupta. Low-jitter on-chip clock for RSFQ circuit applications. Superconductor Science and Technology, 12(11):769-772, 1999.

\section{A The proof of Lemma 4.8}

To compute the second order term $\left(\varepsilon^{2}\right)$ of $\widehat{D}$, we will analyse the $\varepsilon$-dependence of $\widehat{U}^{ \pm}(\xi, \varepsilon):=$ $\widehat{U}^{ \pm}\left(\xi, \mathrm{i} \omega_{0}, \varepsilon\right)$. First we write

$$
\widehat{U}^{ \pm}(\xi, \varepsilon)=e^{\mu_{d}\left(\varepsilon^{2}\right) \xi}\left(\eta_{d}\left(\varepsilon^{2}\right)+V^{ \pm}(\xi, \varepsilon)\right) .
$$

It is easy to verify that $V^{ \pm}(\xi, \varepsilon)$ satisfies

$$
V_{\xi}^{ \pm}(\xi, \varepsilon)=\left(\widehat{\mathbf{A}}\left(\xi, \mathrm{i} \omega_{0}, \varepsilon\right)-\mu_{d}\left(\varepsilon^{2}\right) I\right) V^{ \pm}(\xi, \varepsilon)+R(\xi, \varepsilon) \eta_{d}\left(\varepsilon^{2}\right) \quad \text { and } \quad \lim _{\xi \rightarrow \pm \infty} V^{ \pm}(\xi, \varepsilon)=0
$$

where

$$
R(\xi, \varepsilon)=\widehat{\mathbf{A}}\left(\xi, \mathrm{i} \omega_{0}, \varepsilon\right)-\widehat{\mathbf{A}}_{0}\left(\mathrm{i} \omega_{0}, \varepsilon\right)=\left(\begin{array}{cc}
0 & 0 \\
\cos \left(u_{\varepsilon}(\xi)\right)-\sqrt{1-\varepsilon^{2} \gamma^{2}} & 0
\end{array}\right) .
$$

Hence $R(\xi, \varepsilon) \eta_{d}\left(\varepsilon^{2}\right)$ is vanishing exponentially fast at $\xi= \pm \infty$.

With the symmetry relation (4.2), it follows that

$$
V^{-}(\xi, \varepsilon)=\overline{S V^{+}(-\xi,-\varepsilon)}
$$

hence it is sufficient to determine an expansion for $V^{+}$only. We write

$$
V^{+}(\xi, \varepsilon)=V_{0}(\xi)+\varepsilon V_{1}(\xi)+\varepsilon^{2} V_{2}(\xi)+\ldots
$$


Then $V_{i}(\xi)$ satisfies the following inhomogeneous equation

$$
\frac{\mathrm{d}}{\mathrm{d} \xi} V_{i}=\left(\widehat{\mathbf{A}}\left(\xi, \mathrm{i} \omega_{0}, 0\right)+\mathrm{i} c_{0} I\right) V_{i}+H_{i}(\xi)
$$

since $\mu_{d}(0)=-\mathrm{i} c_{0}$ and where

$$
\begin{aligned}
H_{i}(\xi)= & \left.\sum_{j=1}^{i} \frac{1}{j !} \frac{\partial^{j}}{\partial \varepsilon^{j}}\right|_{\varepsilon=0}\left[\widehat{\mathbf{A}}\left(\xi, \mathrm{i} \omega_{0}, \varepsilon\right)-\mu_{d}\left(\varepsilon^{2}\right) I\right] V_{i-j}(\xi) \\
& +\left(0,\left.\frac{1}{i !} \frac{\partial^{i}}{\partial \varepsilon^{i}}\right|_{\varepsilon=0}\left[\cos \left(u_{\varepsilon}(\xi)\right)-\sqrt{1-\varepsilon^{2} \gamma^{2}}\right]\right)^{T} .
\end{aligned}
$$

The boundary condition is $\lim _{\xi \rightarrow \infty} V_{i}(\xi)=0$. Note that the homogeneous part of this equation does not have an exponential dichotomy. Instead it will be shown that the inhomogeneous part decays exponentially fast to zero for $\xi \rightarrow \infty$. A solution matrix for the system $V_{\xi}=$ $\left[\widehat{\mathbf{A}}\left(\xi, \mathrm{i} \omega_{0}, 0\right)+\mathrm{i} c_{0} I\right] V$ is given by

$$
\chi(\xi)=\left(\begin{array}{cc}
\tanh \xi & \xi \tanh \xi-1 \\
\frac{\mathrm{d}}{\mathrm{d} \xi}(\tanh \xi)-\mathrm{i} c_{0} \tanh \xi & \frac{\mathrm{d}}{\mathrm{d} \xi}(\xi \tanh \xi-1)-\mathrm{i} c_{0}(\xi \tanh \xi-1)
\end{array}\right)
$$

(3.10), hence the evolution matrix for this system is $\Phi(\xi, \tau)=\chi(\xi) \chi(\tau)^{-1}$. So we can conclude that the general solution of (A.2) is given by

$$
V_{i}\left(\xi ; \tau, V_{i}^{0}\right)=\Phi(\xi, \tau) V_{i}^{0}+\int_{\tau}^{\xi} \Phi(\xi, s) H_{i}(s) d s .
$$

It can be shown that $\Phi(\xi, \tau)$ grows at most linear in $|\xi-\tau|$, i.e, there is some constant $\widehat{K}$ such that $|\Phi(\xi, \tau)| \leq \widehat{K}|\xi-\tau|$. Hence if $H_{i}(s)$ is exponentially decaying, then $\int_{\infty}^{\xi} \Phi(\xi, s) H_{i}(s) d s$ is well defined and exponentially decaying in $\xi$. The travelling wave solution $u_{\varepsilon}(\xi)$ decays exponentially fast to $\sqrt{1-\varepsilon^{2} \gamma^{2}}$, hence we see immediately that $\left.\frac{\partial^{i}}{\partial \varepsilon^{i}}\right|_{\varepsilon=0}\left[\cos \left(u_{\varepsilon}(\xi)\right)-\sqrt{1-\varepsilon^{2} \gamma^{2}}\right]$ is exponentially decaying for any $i$. Hence $H_{0}(s)$ is exponentially decaying and with the boundary condition $V_{0}(\xi) \rightarrow 0$ as $\xi \rightarrow \infty$, it follows that $V_{0}(\xi)=\int_{\infty}^{\xi} \Phi(\xi, s) H_{0}(s) d s$. This function is exponentially decaying too. With an iteration argument it follows that

$$
V_{i}(\xi)=\int_{\infty}^{\xi} \Phi(\xi, s) H_{i}(s) d s
$$

and that $V_{i}(\xi)$ is exponentially decaying at $\xi \rightarrow \infty$. It follows from the expression (A.3) that,

$$
V_{0}(\xi)=\int_{\infty}^{\xi} \Phi(\xi, s)\left(\begin{array}{c}
0 \\
\cos \left(u_{0}(s)\right)-1
\end{array}\right) d s=\left(\begin{array}{c}
\tanh \xi-1 \\
\operatorname{sech}^{2} \xi-\mathrm{i} c_{0}(\tanh \xi-1)
\end{array}\right) .
$$

This expression is consistent with (3.10) and rephrases the relation (4.3). Next look at $V_{1}(\xi)$. First we observe that

$$
H_{1}(\xi)=\left(\begin{array}{c}
0 \\
-u_{1}(\xi) \sin \left(u_{0}(\xi)\right)\left[1+V_{0}(\xi)[1]\right]
\end{array}\right)=\left(\begin{array}{c}
0 \\
-u_{1}(\xi) \tanh \xi \sin \left(u_{0}(\xi)\right)
\end{array}\right),
$$


where $u_{1}(\xi)=\left.\frac{\partial}{\partial \varepsilon}\right|_{\varepsilon=0} u_{\varepsilon}(\xi)$, the first order correction to the travelling wave solution. In section 2, we have derived an integral expression for $u_{1}$, hence with (A.3), we have an expression for $V_{1}$, involving a double integral. Note that $u_{1} / \gamma$ does not depend on any of the parameters, hence $V_{1} / \gamma$ does not depend on any of the parameters neither. By expanding $\Phi(\xi, s) H_{1}(s)$, it follows that $\Im\left(V_{1}(\xi)[1]\right)=0$ and $\Im\left(V_{1}(\xi)[2]\right)=-c_{0} \Re\left(V_{1}(\xi)[1]\right)$. For later use, we note that it can be calculated numerically that $V_{1}(0)=\gamma\left(0.63584,0.89923-0.63584 \mathrm{i} c_{0}\right)^{T}$.

For the second order term of the Evans function, we need $\Re\left(V_{2}(0)[1]\right)$ too. First we calculate that

$$
\begin{aligned}
H_{2}(\xi)= & \left(\begin{array}{c}
0 \\
-c_{0} K_{2}(\tanh \xi-1)-\tanh \xi\left[u_{2} \sin \left(u_{0}\right)+\frac{1}{2} u_{1}^{2} \cos \left(u_{0}\right)-\frac{1}{2} \gamma^{2}\right]-u_{1} \sin \left(u_{0}\right) V_{1}[1]
\end{array}\right) \\
& -\mathrm{i} K_{2}\left(\begin{array}{c}
(\tanh \xi-1) \\
-\operatorname{sech}^{2} \xi
\end{array}\right),
\end{aligned}
$$

where $K_{2}=\frac{c_{0}}{8}\left(\alpha^{2}+2 \gamma^{2}\right)-c_{2}$, where $c_{\varepsilon}=c_{0}+\varepsilon^{2} c_{2}+\mathcal{O}\left(\varepsilon^{4}\right)$. With some tedious calculations it follows that

$$
\begin{aligned}
\Re\left(V_{2}(0)[1]\right)=-\int_{0}^{\infty} & \left(\tanh ^{2}(s)\left[u_{2}(s) \sin \left(u_{0}(s)\right)+\frac{1}{2} u_{1}^{2}(s) \cos \left(u_{0}(s)\right)-\frac{1}{2} \gamma^{2}\right]\right. \\
& \left.+\tanh (s) u_{1}(s) \sin \left(u_{0}(s)\right) V_{1}(s)[1]\right) d s
\end{aligned}
$$

With the expressions from section 2, a numerical evaluation of the integrals involved gives $\Re\left(V_{2}(0)[1]\right)=\gamma^{2} 1.1503$.

Finally, we can consider the Evans function. First we observe that

$$
\begin{aligned}
\widehat{D}\left(\mathrm{i} \omega_{0}, \varepsilon\right) & =\widehat{U}^{+}(0, \varepsilon) \wedge \widehat{U}^{-}(0, \varepsilon) e^{-2 \mu_{d}\left(\varepsilon^{2}\right) \xi}=\left[\eta_{d}\left(\varepsilon^{2}\right)+V^{+}(0, \varepsilon)\right] \wedge\left[\eta_{d}\left(\varepsilon^{2}\right)+V^{-}(0, \varepsilon)\right] \\
& =\left[\eta_{d}\left(\varepsilon^{2}\right)+V^{+}\left(0, \varepsilon^{2}\right)\right] \wedge\left[\eta_{d}\left(\varepsilon^{2}\right)+\overline{S V^{+}(0,-\varepsilon)}\right] .
\end{aligned}
$$

We have just derived that up to order $\varepsilon^{3}$

$$
\begin{aligned}
& \eta_{d}\left(\varepsilon^{2}\right)+V^{+}(0, \varepsilon)=\left(\begin{array}{l}
0 \\
1
\end{array}\right)+\varepsilon \gamma\left(\begin{array}{c}
0.63584 \\
0.89923-0.63584 \mathrm{i} c_{0}
\end{array}\right)+\varepsilon^{2} \gamma^{2}\left(\begin{array}{c}
1.1503+\mathrm{i} \Im\left(V_{2}(0)[1]\right) \\
V_{2}(0)[2]
\end{array}\right) \\
& \eta_{d}\left(\varepsilon^{2}\right)+\overline{S V^{+}(0,-\varepsilon)}=\left(\begin{array}{c}
0 \\
-1
\end{array}\right)-\varepsilon \gamma\left(\begin{array}{c}
0.63584 \\
-0.89923-0.63584 \mathrm{i} c_{0}
\end{array}\right)+\varepsilon^{2} \gamma^{2}\left(\begin{array}{c}
1.1503-\mathrm{i} \Im\left(V_{2}(0)[1]\right) \\
-\overline{V_{2}(0)[2]}
\end{array}\right)
\end{aligned}
$$

Hence $\widehat{D}\left(\mathrm{i} \omega_{0}, \varepsilon\right)=-2 \varepsilon^{2} \gamma^{2}(1.1503-0.63584 \cdot 0.89923)+o\left(\varepsilon^{2}\right)=-1.1571 \varepsilon^{2} \gamma^{2}+o\left(\varepsilon^{2}\right)$.

Although $\widehat{D}(\sigma, \varepsilon)$ is not analytic in $\sigma$ near $\sigma=\mathrm{i} \omega_{0}$, it is differentiable as a function of $\sqrt{\sigma-\mathrm{i} \omega_{0}}[8,11]$. Hence we have

$$
\begin{aligned}
\widehat{D}(\sigma, \varepsilon) & =\widehat{D}(\sigma, 0)-1.1571 \gamma^{2} \varepsilon^{2}+\mathcal{O}\left(\varepsilon \sqrt{\sigma-\mathrm{i} \omega_{0}}+\varepsilon^{3}\right) \\
& =-2(1+\mathrm{i})\left(1-c_{0}\right)^{-\frac{1}{4}} \sqrt{\sigma-\mathrm{i} \omega_{0}}-1.1571 \gamma^{2} \varepsilon^{2}+\mathcal{O}\left(\varepsilon \sqrt{\sigma-\mathrm{i} \omega_{0}}+\left(\sqrt{\sigma-\mathrm{i} \omega_{0}}\right)^{2}+\varepsilon^{3}\right)
\end{aligned}
$$

Since $\arg \left(2(1+\mathrm{i})\left(1-c_{0}\right)^{-\frac{1}{4}} \sqrt{\sigma-\mathrm{i} \omega_{0}}\right) \in\left(-\frac{\pi}{4}, \frac{3 \pi}{4}\right)$ and $\arg \left(1.1571 \gamma^{2} \varepsilon^{2}\right)=0$, we conclude that $\widehat{D}(\sigma, \varepsilon)$ cannot have eigenvalues near $\sigma=\mathrm{i} \omega_{0}(\widehat{D}(\sigma, \varepsilon)$ has zeroes on another sheet of the associated Riemann surface $\mathcal{R}$, see Remark 5.7). 